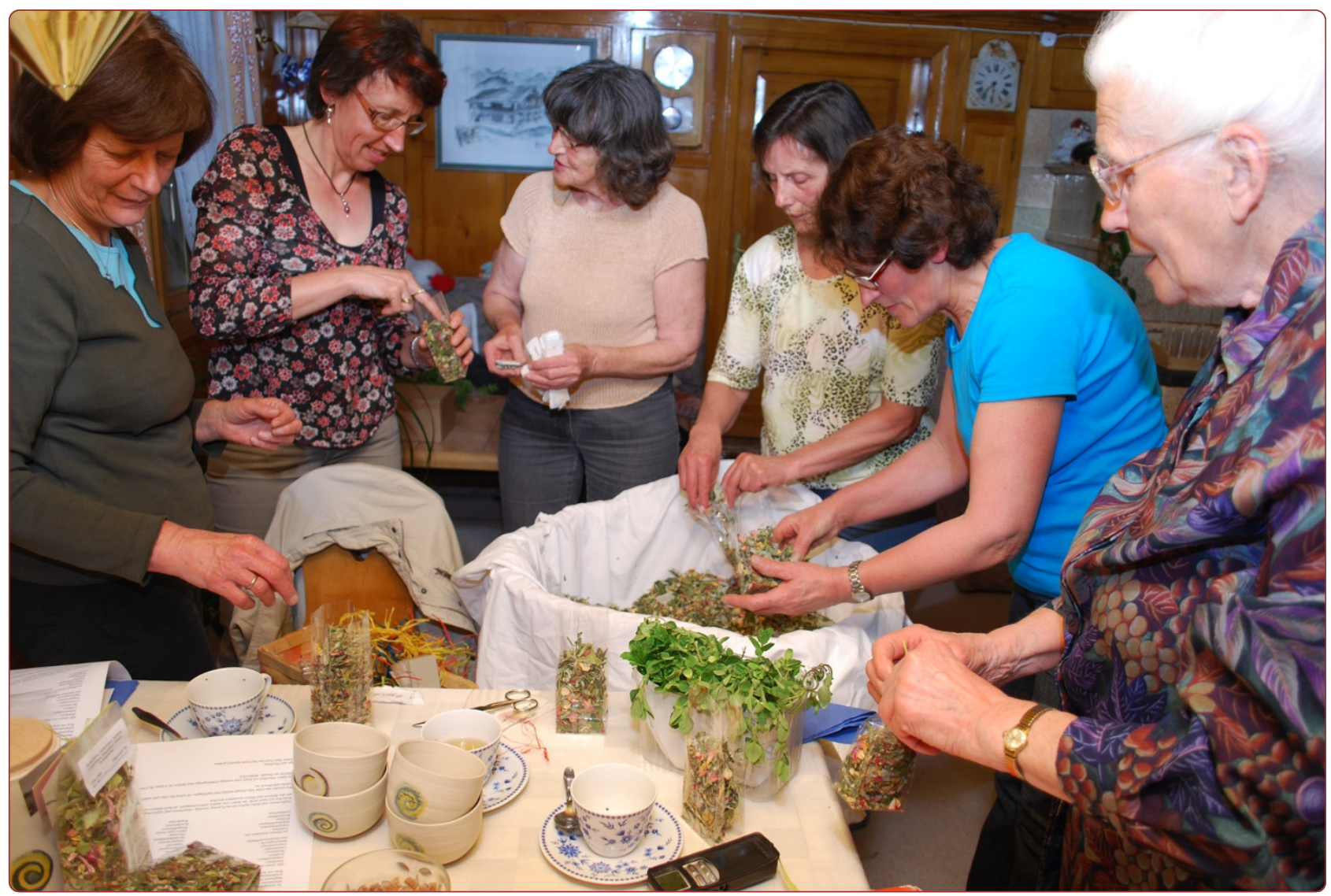

\title{
Gathering "tea" - from necessity to connectedness with nature. Local knowledge about wild plant gathering in the Biosphere Reserve Grosses Walsertal (Austria)
}

Grasser et al. 


\title{
Gathering "tea" - from necessity to connectedness with nature. Local knowledge about wild plant gathering in the Biosphere Reserve Grosses Walsertal (Austria)
}

\author{
Susanne Grasser*, Christoph Schunko and Christian R Vogl
}

\begin{abstract}
Background: Wild plant gathering is an essential element in livelihood strategies all over the world. However due to changing circumstances in Europe, the reason for gathering has altered from one of necessity in the past to a pleasurable activity today. Wild plant gathering has therefore also received renewed attention as a form of intangible cultural heritage expressing local preferences, habits and man's relationship with nature. In the Biosphere Reserve Grosses Walsertal (Austria), local people's knowledge of the gathering of wild plants and their perception of their own gathering activities are being documented. The focus of this paper is on the uses of herbal teas and the informal guidelines for gathering plants that have been issued by the Bergtee (mountain tea) association.
\end{abstract}

Methods: Thirty-six free-list interviews were conducted with subsequent semi-structured interviews and three focus group meetings held with members of the Bergtee association. Participatory observation (gathering and processing plants, mixing and marketing tea) also allowed for greater understanding of what had been reported.

Results: In total, 140 different gathered plant species were listed by respondents. Herbal tea is the most frequently mentioned use. The Bergtee association, founded by a young man and two middle-aged women in the valley, is a good example of the link between biological and cultural diversity, with the aim of sharing the biosphere reserve's natural treasures as well as local plant-related knowledge in the form of herbal tea products. The association's informal guidelines for gathering reflect people's attitude to nature: monetary income does not play a major role in gathering plants; instead people's appreciation of the value of the nature around them is to the fore.

Conclusions: Gathering wild plants can be seen as an expression of people's regional identity. The conscious appreciation of nature and related local knowledge is crucial for the sustainable conservation and use of the Biosphere Reserve's resources.

Keywords: Local knowledge, Herbal tea, Wild plant gathering, Regional identity, Revitalisation of tradition

\footnotetext{
* Correspondence: susanne.grasser@boku.ac.at

Working Group Knowledge Systems and Innovations, Division of Organic Farming, Department for Sustainable Agricultural Systems, University of Natural Resources and Life Sciences (BOKU), Gregor-Mendel Straße 33, Vienna 1180, Austria
}

\section{Biomed Central}

(c) 2012 Grasser et al.; licensee BioMed Central Ltd. This is an Open Access article distributed under the terms of the Creative Commons Attribution License (http://creativecommons.org/licenses/by/2.0), which permits unrestricted use, distribution, and reproduction in any medium, provided the original work is properly cited. 


\section{Background}

The gathering of wild plants is an essential element in livelihood strategies all over the world [1,2]. The focus of European ethnobotanical studies on gathering has been on study areas in the Mediterranean region such as Turkey [3], Greece [4], Italy [5,6], Spain and Portugal [7-9], Cyprus [10], Serbia [11], Montenegro [12], Kosovo [13], Bosnia and Herzegovina [14,15]. However there are also a few studies in Central Europe, including Germany [16], Austria [17,18], Switzerland [19] and Poland [20]. These study areas all share a historical shift from agriculture to wage labour which reduced gathering opportunities and activities $[3,17,21,22]$. Up to the period after the Second World War, people depended on natural resources, and wild plant gathering was an important supplement to daily nutrition in a culture of subsistence $[8,18,20]$ as well as for medicinal needs $[17,22]$. Nowadays, in many countries the gathering of wild plants is no longer a necessity [22,23].

As local knowledge about wild gathered plant species and their uses is under severe threat as a result of economic globalisation, there is an urgent need to document and safeguard it [24,25], especially in remote areas where this kind of development has not yet led to a complete loss of traditions [26]. It is important to establish a record like this before local knowledge vanishes for good along with the current generation of elderly people [25].

Although local knowledge is vanishing with the elderly, at the same time an interest in this topic can be observed among young and middle-aged people [27]. In a way, plant gathering is becoming fashionable. There are many popular herb books [28-31] focusing on plant species that can be gathered, as well as seminars and courses on plants how to process and use them [17]. In Poland, for example, media publications have led to an increase in the culinary use of some wild plants [20]. In the 1980s, local food producers started to popularise, rediscover and even invent "local products" for selling to tourists [20]. Nowadays, "consumption is determined less by calorie input and more by the pleasure of gathering from the wild, recreating traditional practices and enjoying characteristic flavours" [8].

Wild plant gathering has also received renewed attention as a form of intangible cultural heritage [27,32-35]. This activity is one example of the inextricable link between biodiversity and cultural diversity [36] and reflects symbols of local identities [33]. It is an irreplaceable part of the cultural history of a region $[18,37,38]$ and therefore an expression of people's local identity and traditions [24,39].

Biosphere reserves are places of high biodiversity and increased awareness of cultural values and identity [40-42]. Their credo is "to use nature without harming it" [43].
In the mission statement of the Biosphere Reserve Grosses Walsertal, the main aim is to raise people's awareness of cultural values and natural resources as the main basis of livelihood for people in the valley (literally translated from [41]).

It is of interest to see which plant species are gathered and how gathering is perceived by local people who carry out this activity in Grosses Walsertal - the study area being a biosphere reserve and also part of the, in economic terms, highly-developed Austrian province of Vorarlberg. Is gathering vanishing or at risk of being lost? Why do people gather plants today? What knowledge is involved and what is its current image? One association - the Bergtee (literally: mountain tea) - dealing with plants and plant knowledge in the Biosphere Reserve Grosses Walsertal has been selected as being representative of local people's attitudes towards plant gathering. This paper therefore focuses on herbal tea as one example of plant use.

\section{Methods \\ Field site}

Grosses Walsertal is an alpine valley in the centre of Vorarlberg, the westernmost province of Austria (Figure 1). It is a west-southwest, east-northeast-oriented, gorgelike valley without a distinct valley floor, formed by the river Lutz. Covering an area of 192 square kilometers and with a population of 3,400 , the valley is very sparsely populated. The dispersed settlements in the valley are mostly situated on the northern (sunny) side. These are the villages of Thüringerberg (880 $\mathrm{m}$ a.s.l.), St. Gerold (848 $\mathrm{m}$ a.s.l.), Blons (903 $\mathrm{m}$ a.s.l.), Sonntag (875 m a.s.l.) and Fontanella (1,145 $\mathrm{m}$ a.s.l.). On the southern (shady) side are Raggal (1,015 m a.s.l.) and Marul (976 m a.s.l.) [43].

Geologically, Grosses Walsertal is divided into two: the northern part is distinguished by the gentle green mountains of Flysch and the southern part by hills belonging to the Kalkhochalpen (limestone layers). Its altitude ranges from 580 to 2,704 $\mathrm{m}$ a.s.l. [43].

The climate in Vorarlberg is typical of central Europe, but relatively cool and with higher precipitation [44]. In Grosses Walsertal, depending on exposure to the sun and altitude, local temperatures and precipitations can vary greatly. The mean annual temperature is $6.7^{\circ} \mathrm{C}$ (measured at an altitude of 1,140 $\mathrm{m}$ a.s.l.). Annual precipitation varies from $1,791 \mathrm{~mm}$ per year (at $900 \mathrm{~m}$ a.s.l. in Blons) to $1,883 \mathrm{~mm}$ per year (at 1,140 $\mathrm{m}$ a.s.l. in Fontanella) [45]. The vegetation period across the settlement area of the valley is between 180 and 240 days, again depending on altitude [46]. Due to its geological and morphological variety, the valley is very rich in species [44].

Grosses Walsertal is named after people who emigrated from the Swiss Wallis region in the $13^{\text {th }}$ century. 


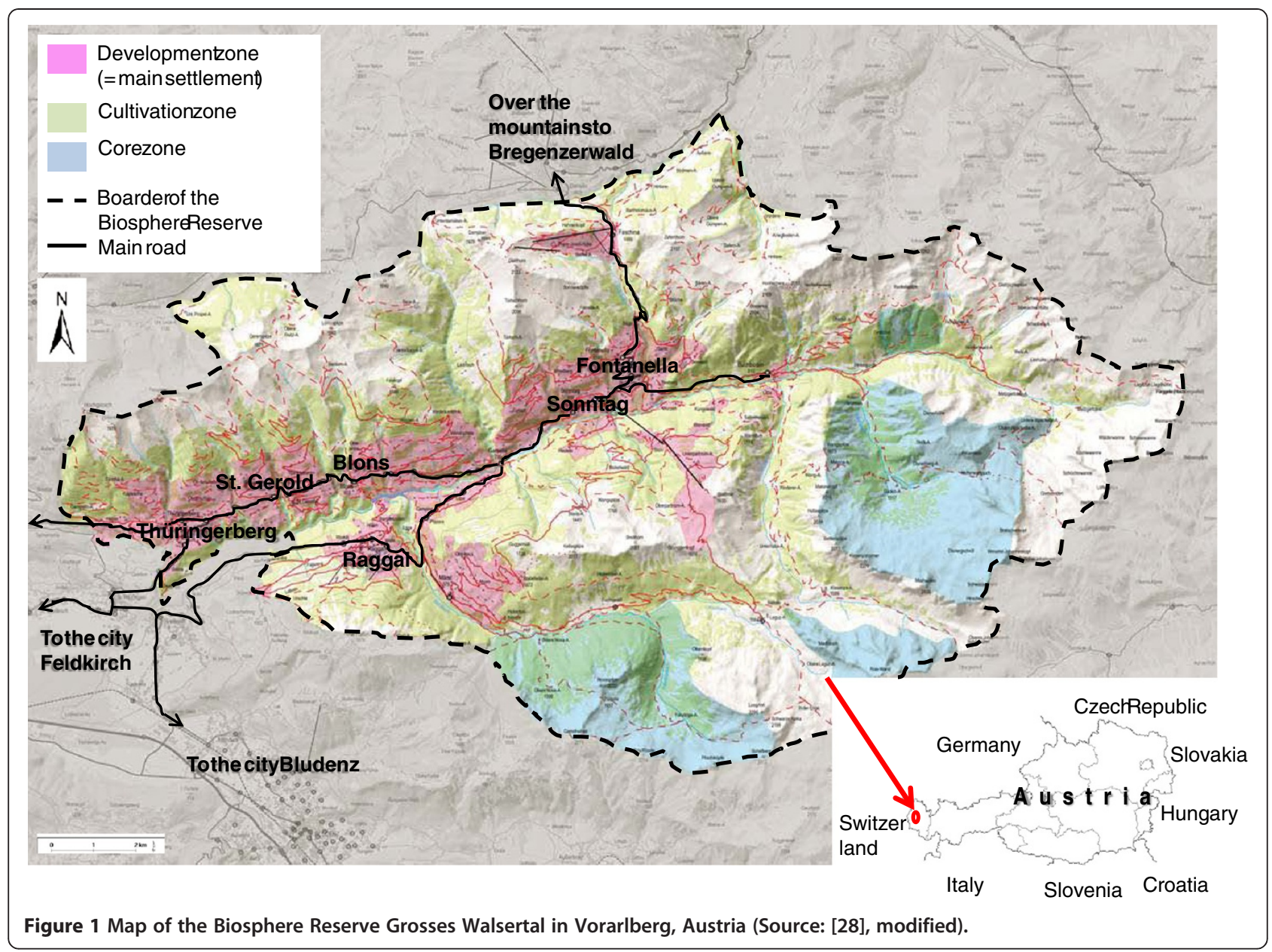

Prior to this, the outer part of the valley had already been inhabited by Rhaeto-Romanic hunters and farmers. The Walser people lived as free peasants and controlled the passes and borders. For a long time, keeping animals for milk and meat formed the sole basis of their existence. Arable farming existed only at self-sufficiency level [44]. One type of agriculture is characteristic of Grosses Walsertal: the Staffel-/Mehrstufenwirtschaft, a specific kind of transhumance, i.e. as the year progresses, farmers move with their livestock (and, especially in the past, with their entire household) to different sites at different elevations: the home farm (winter), the Maisäß (elevation 1,300 - 1,700 $\mathrm{m}$ a.s.l., spring and autumn) and the Alp (elevation $>1,700 \mathrm{~m}$ a.s.l., summer) [47].

Due to their remoteness, poverty and subsistence up to the end of the Second World War, Walser people used to rely on their local natural resources [48]. For centuries, the people of this remote region were almost cut off entirely. This situation has left its mark: to this day the Walsertal prides itself on a culture and dialect of its own [44].

Gathering plants seems to have a "history" in Grosses Walsertal, even though there is no literature exclusively from this valley to confirm it. Old literature from other nearby regions might also be valid for Grosses Walsertal (e.g. from the Montafon [49] or from Switzerland [50]). The botany and local people's uses of plants in the district of Vorarlberg were documented by Schertler [30], for example, in a popular book full of pictures, stories and recipes.

Local plant knowledge was probably passed on orally or people might have kept handwritten booklets at home. A collection in the Walser dialect was only published in 1996 [51]. This is a collection of statements by local people about plants and other homemade remedies for medicinal purposes. Being part of people's farming activity, gathering plants was not perceived as something special or extraordinary. However farming and with it the culture of subsistence has changed.

There are now 180 farms in the area, $40 \%$ of which are organic [43]. This provides $11.3 \%$ of employment, whereas $16.1 \%$ of people work in small skilled trade enterprises, $7.7 \%$ in tourism and $3.4 \%$ in public services. $61.5 \%$ have to commute out of the valley for work [52]. Grosses Walsertal became a commuter region from the 1970s onwards [52]. 
Grosses Walsertal has always been recognised as the poorest part of Vorarlberg [53]. After a varied history, including a huge avalanche disaster in 1954 and the rise of tourism since the 1960s, the valley's inhabitants were confronted with reorientation and repositioning in the mid-1990s. A strategy was required to give the region a new perspective on economic development [53]. In 2000 Grosses Walsertal was acknowledged as a UNESCO Biosphere Reserve. This led to it being structured into four zones: the core zone (20.9\% of the area), the cultivation zone $(64.4 \%$ of the area), the development zone $(13.7 \%$ of the area) and the regeneration zone (1.0\% of the area) [54].

The Biosphere Reserve's mission statement declares its aim as being to "raise consciousness of cultural values and natural resources as the main means of livelihood for people in Grosses Walsertal". One of its main principles is "to be in awe of God and nature and appreciate people's dignity... A responsible and sparing acquaintance with energy and resources is important to all inhabitants." (literally translated from [41]). In relation to plant diversity, there are two associations dealing with herbs: the Bergtee and Alchemilla. The women in these associations use their range of products and services to demonstrate the value of the varied cultural landscape and make an important contribution to adding value in the region [43].

\section{Free lists, semi-structured interviews, participatory observation}

Between July and September 2008, thirty-six interviews were conducted in all six municipalities using free lists and subsequent semi-structured interviews [55,56]. After being given a short introduction to this research project, respondents were asked to list all the plant species gathered in the wild which they know, using the free-listing question: "Welche Pflanzen fallen dir ein, die hier im Tal wild wachsen und gesammelt werden?" (literal translation: "What plants can you think of which grow wild in the valley and are gathered?"). In some cases it was necessary to ask further questions (e.g. "If you think about the seasons ..."), but without ever offering examples of plants so as not to influence the respondents' answers. When respondents started to run out of ideas, the plants already listed were repeated and respondents asked whether anything else came to mind.

Sampling of respondents was done in the form of snowball sampling [56]. The starting points were members of the Bergtee and Alchemilla plant associations. In total, 36 respondents, two male and 34 female aged between 27 and 89 years old (average age: 60), were interviewed. Nine of the respondents are involved in the Bergtee project and nine in the Alchemilla project. Half of the respondents are not involved in any plant-related project, but represent "common" local knowledge about gathering plants in the valley. Twenty-three of the respondents currently run a farm.

A clear expression of consent was requested before each interview. Where applicable, ethical guidelines issued by the International Society of Ethnobiology [57] were followed carefully.

Field notes were taken during the interviews to record the information given and brain protocols completed afterwards. The interviews were also recorded using a digital voice recorder. Photographs of plants and of people gathering and processing plants were taken to provide visual documentation of the information given by local people. Voucher specimens of all plants available during field research and growing in the wild are stored in the herbarium of the University of Natural Resources and Life Sciences in Vienna. Cultivated plants in people's gardens were not taken as voucher specimens. Plant identification is based on Flora Helvetica [58].

Three meetings were arranged for data collection (focus groups adapted by the authors) with the women involved in the Bergtee project: one meeting with women from Raggal, one with women from Thüringerberg, St. Gerold and Blons and one with women from Sonntag and Fontanella, always attended by one or both project leaders. These meetings, usually held by the Bergtee association once a year, are used to exchange experiences of plant gathering and use, but also for the leaders to give news of the Bergtee project and raise awareness of gathering guidelines. In this case, in 2010, data collection for this project was integrated into the meeting.

Participatory observation was conducted during the whole field research, including plant gathering with local people, helping with agricultural activities (e.g. hay making) or processing plants (e.g. tea mixing, cooking and ointment or soap making) as well as participating in everyday life and customary festivities (e.g. Easter customs, thanksgiving).

An Access database was set up to organise and store the information gathered. Data analysis was carried out in MS Access [59], MS Excel [60] and Anthropac [61]. The interviews were partly transcribed using Express scribe v5.00 [62] and then coded for qualitative analysis.

\section{Results}

First a brief overview is given of the plants reported in the free-list interviews, followed by people's associations to explain how these listed plants came to be mentioned. As tea is the most frequently mentioned use report, the focus was on the Bergtee (mountain tea) association as a very good example of the inextricable link between nature and culture (biocultural diversity; [36]). Contact with this association allowed their informal guidelines for plant gathering, which are only transmitted orally, to 
be documented to show local people's attitude towards this activity as well as their relationship with nature and natural resource management. This is related to local people's motivation for plant gathering which has changed over time. The reasons for gathering plants complete the results section with the question "Gathering wild plants - a revitalisation of tradition?" and provide an idea of what prospects might be like in future.

\section{Gathering wild plants - which plants and why?}

The respondents mentioned 892 plant species in all when asked which plants were known to be gathered in the wild in the valley. These reports refer to 140 different plant species belonging to 49 different plant families (families with the most use reports: Lamiaceae with 20 plant species followed by Asteraceae with 16 and Rosaceae with 12). Each respondent listed between 10 and 50 plant species (average: 25 plant species; standard deviation: 8.7) (Figure 2)

The twenty most frequently listed plant species are Alchemilla alpina, Alchemilla vulgaris L. agg., Calendula officinalis L., Sambucus nigra L., Hypericum perforatum L., Achillea millefolium agg., Rhododendron sp., Urtica dioica L., Rubus idaeus L., Mentha sp., Primula veris L./Primula elatior (L.)L., Arnica montana L., Plantago lanceolata L., Matricaria chamomilla L., Picea abies (L.)H. KARST./Abies alba MILL., Salvia officinalis L., Thymus serphyllum L. agg., Trifolium pratense L.,
Vaccinium myrtillus L. and Taraxacum officinale agg (Table 1). Each of these twenty plant species was listed by at least 44 percent of respondents.

There is no statistically significant difference in knowledge between the people involved in the Bergtee association, those involved in Alchemilla and "ordinary" people without any involvement in these herb associations.

The respondents were only asked to list plants, but spontaneously many respondents also mentioned what they use some of these plants for (in total 774 use reports). These use reports were subsequently assigned by the researcher to the following categories: nutrition (NUT), medicinal application (MED), veterinary application (VET), utilisation for decoration (DEC) and other uses (OTH) (Figure 3). The nutrition (NUT) category comprises plants used for eating - e.g. eaten raw in salads or added to cakes, biscuits, bread, omelettes, soups, cooked as "spinach" or processed as jam, "honey" (a very thick syrup) or "pesto". It also includes plants used for drinking, e.g. dried for herbal tea, in the form of syrup and juice or added to punch and mixed with alcohol such as liqueur and schnapps or distilled. The medicinal (MED) category includes application forms such as herbal teas for drinking (internal) or bathing (external), oils, ointments for grease and schnapps for rubbing in, but also non-processed raw plants with healing effects (e.g. to be eaten or applied fresh on wounds). The veterinary medicine (VET) category includes all medicinal

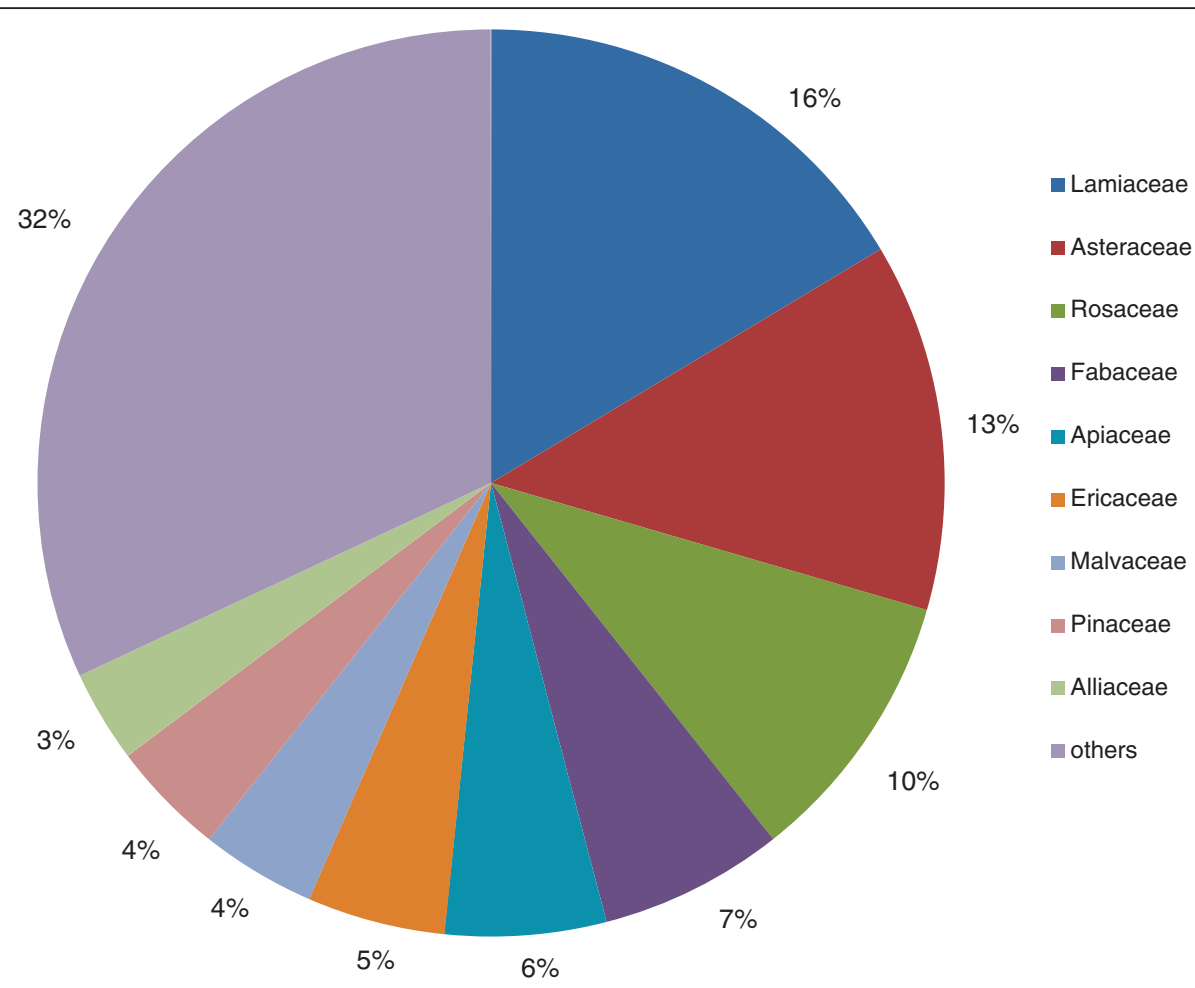

Figure 2 Most quoted plant families in \%, frequency of mentions within one plant family $>3$ ( $f<3$ are summarised as "others") $(n=36)$. 
Table 1 The most frequently listed plant species gathered in the wild at the Biosphere Reserve Grosses Walsertal (free lists, $n=36$, frequency $>6$ ). $w=$ growing in the wild, $c=$ cultivated in the garden

\begin{tabular}{|c|c|c|c|c|c|c|}
\hline Scientific name & German name & Family & Frequency & Mean rank & Smith's S & Status ${ }^{*}$ \\
\hline Alchemilla alpina L. agg. & Silbermantel & Rosaceae & 30 & 8.2 & 0.58 & w \\
\hline Alchemilla vulgaris L. agg. & Frauenmantel & Rosaceae & 30 & 8.8 & 0.57 & w \\
\hline Calendula officinalis L.* & Ringelblume & Asteraceae & 29 & 10.6 & 0.49 & c \\
\hline Sambucus nigra $\mathrm{L}$. & Schwarzer Holunder & Caprifoliaceae & 29 & 13.9 & 0.38 & $w, c$ \\
\hline Achillea millefolium L. & Schafgarbe & Asteraceae & 28 & 10.8 & 0.47 & w \\
\hline Hypericum perforatum L. & Johanniskraut & Hyperiaceae & 28 & 11.8 & 0.46 & $w, c$ \\
\hline Rhododendron sp.** & Alpenrose & Ericaceae & 26 & 11.3 & 0.41 & w \\
\hline Urtica dioica L. & Brennnessel & Urticaceae & 23 & 11.3 & 0.38 & w \\
\hline Rubus idaeus L.* & Himbeere & Rosaceae & 22 & 15.2 & 0.30 & $w, c$ \\
\hline Mentha sp. * & Pfefferminze & Lamiaceae & 21 & 10.7 & 0.37 & c \\
\hline Plantago lanceolata $\mathrm{L}$. & Spitzwegerich & Plantaginaceae & 20 & 10.3 & 0.35 & w \\
\hline Arnica montana L. & Arnika & Asteraceae & 20 & 10.4 & 0.35 & w \\
\hline Primula veris L./Primula elatior (L.) L.**** & Schlüsselblume & Primulaceae & 20 & 12.0 & 0.34 & w \\
\hline Abies alba MILL./Picea abies (L.) H. KARST.***** & Tanne & Pinaceae & 19 & 12.9 & 0.31 & w \\
\hline Matricaria chamomilla L.* & Kamille & Asteraceae & 19 & 15.7 & 0.23 & c \\
\hline Thymus serphyllum L. agg. & Wilder Thymian & Lamiaceae & 18 & 12.7 & 0.27 & w \\
\hline Salvia officinalis L.* & Salbei & Lamiaceae & 18 & 14.6 & 0.22 & c \\
\hline Trifolium pratense $\mathrm{L}$. & Rotklee & Fabaceae & 17 & 12.5 & 0.26 & w \\
\hline Taraxacum officinale WEBER & Löwenzahn & Asteraceae & 16 & 12.4 & 0.28 & w \\
\hline Vaccinium myrtillis L. & Heidelbeere & Ericaceae & 16 & 17.2 & 0.16 & w \\
\hline Melissa officinalis L.* & Zitronen Melisse & Lamiaceae & 15 & 12.5 & 0.20 & c \\
\hline Fragaria vesca $\mathrm{L}$. & Erdbeere & Rosaceae & 15 & 16.2 & 0.20 & w \\
\hline Malva neglecta WALLR. & Käspappel & Malvaceae & 15 & 18.5 & 0.17 & w \\
\hline Tussilago farfara $\mathrm{L}$. & Huflattich & Asteraceae & 14 & 12.9 & 0.21 & w \\
\hline Juniperus communis L. & Wacholder & Cupressaceae & 14 & 13.7 & 0.19 & w \\
\hline Equisetum arvense L. & Acker Schachtelhalm & Equisetaceae & 14 & 15.7 & 0.20 & w \\
\hline Tilia sp. & Linde & Tiliaceae & 14 & 17.4 & 0.16 & $w, c$ \\
\hline Bellis perennis $\mathrm{L}$. & Gänseblümchen & Asteraceae & 13 & 12.1 & 0.23 & w \\
\hline Rubus fruticosus L. agg. & Brombeere & Rosaceae & 12 & 13.8 & 0.18 & $w, c$ \\
\hline Ribes nigrum L.* & Schwarze Johannisbeere & Grossulariaceae & 11 & 13.5 & 0.15 & c \\
\hline Peucedanum ostruthium (L.) W.D.J.KOCH & Meisterwurz & Apiaceae & 10 & 14.0 & 0.15 & w \\
\hline Artemisium absinthum L. & Wermut & Asteraceae & 10 & 15.1 & 0.13 & $w, c$ \\
\hline Trifolium repens $L .{ }^{* * *}$ & Weissklee & Fabaceae & 9 & 14.4 & 0.11 & w \\
\hline Origanum vulgare $\mathrm{L}$. & Wilder Majoran & Lamiaceae & 8 & 13.1 & 0.13 & w \\
\hline Sambucus racemosa $\mathrm{L}$. & Roter Holunder & Caprifoliaceae & 8 & 18.1 & 0.09 & w \\
\hline Pinus mugo TURRA & Latsche & Pinaceae & 8 & 20.0 & 0.09 & w \\
\hline Euphrasia officinalis L.p.p. & Augentrost & Scrophulariaceae & 8 & 20.0 & 0.07 & w \\
\hline Rosmarinus officinalis L.* & Rosmarin & Lamiaceae & 8 & 20.5 & 0.08 & c \\
\hline Rosa sp.* & Rose & Rosaceae & 7 & 14.8 & 0.09 & c \\
\hline Monarda didyma $\mathrm{L}^{*}$ & Gold Melisse & Lamiaceae & 7 & 16.8 & 0.06 & c \\
\hline
\end{tabular}


Table 1 The most frequently listed plant species gathered in the wild at the Biosphere Reserve Grosses Walsertal (free lists, $\mathbf{n}=36$, frequency $>6$ ). $\mathbf{w}=$ growing in the wild, $\mathbf{c}=$ cultivated in the garden (Continued)

\begin{tabular}{llccc}
\hline Symphytum officinale L. & Beinwell & Boraginaceae & 7 & 18.1 \\
Lavandula officinalis Chaix, L. spica L., L. vera* & Lavendel & Lamiaceae & 7 & 20.0 \\
\hline
\end{tabular}

* Respondents were asked for "wild species gathered". Nevertheless respondents also mention species i) that used to be gathered in the wild and are now grown in gardens and ii) cultivated species.

** Under the term Rhododendron sp., Rh. ferrugineum and Rh. hirsutum are sub-summarised - respondents do not seem to distinguish between these two species in their local name "Alpenrose". Some respondents use it as the generic term, for some they are just the same plant. Only a few would distinguish between Rhodedendron ferrugineum and call it "Alpenrose" and Rhododendron hirsutum and call it "Steinrösli".

*** The local name "Weißklee" is translated with the scientific name Trifolium repens. Under this term, T. montanum, T. thalii, T. hybridum are sub-summarised as respondents do not distinguish between the white flowering clover plants.

**** Only a few respondents distinguished between Primula elatior (which they call "Schlüsselblume") and Primula veris (which they call "Himmelschlüssel"). Usually, the local name "Schlüsselblume" and "Himmelschlüssel" were used synonymously.

***** The local name "Tanne" can be either Abies alba ("Weißtanne") or Picea abies ("Rottanne"). Some respondents distinguish between these two species; others refer to both plant species using the term "Tanne".

plants also used in veterinary folk medicine as homemade remedies (and most likely also mentioned for humans).

In the decoration (DEC) category the so-called "Alpabtrieb" was mentioned most frequently as a use report. This is a farming tradition and common festivity to celebrate a successful summer on the alpine pasture. Livestock (mainly cattle) and herders are decorated with rosemary (Rosmarinus officinalis) and carnations (Dianthus sp.), combined with branches of fir (Abies alba MILL.) or spruce (Picea abies (L.)H.KARST.). Juniper (Juniperus communis L.) and Rhododendron sp. are also used for decoration. During the Alpabtrieb on one specific day in autumn, people and livestock decorated in this way move down from the alpine pastures in the higher regions to their homes.

A few other use reports in this category are the decoration of dishes with nice flowers or leaves. Other uses $(\mathrm{OTH})$ are for fertilizer, insecticides, incense for fumigation or use in herb pillows for example.

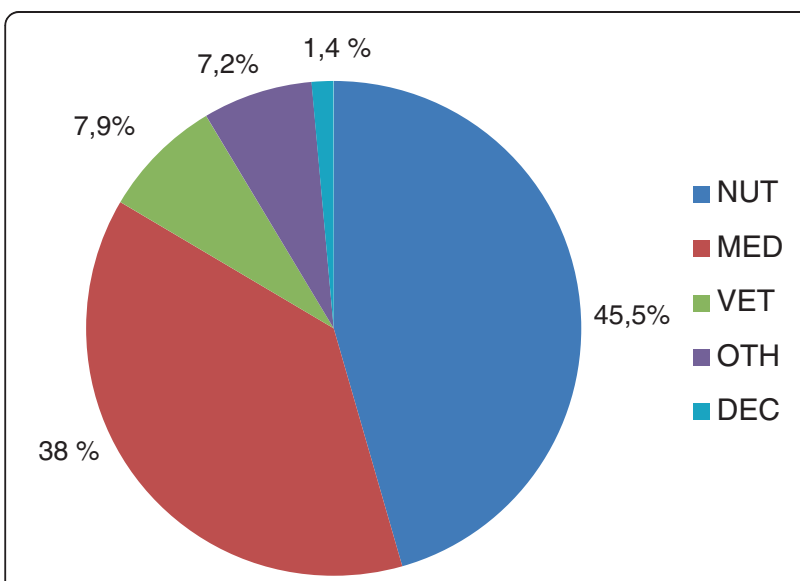

Figure 3 Number of use reports in \% sorted by use categories (NUT - nutrition, MED - medicinal, VET - veterinary, DEC - decoration, OTH - others) $(n=36)$.
A brief description of uses, applications and preparations of all plant species named by more than two respondents shows the diversity of uses practiced by local people in Grosses Walsertal (Table 2). Most of these uses refer to nutritional purposes (NUT category) and plants used for drinking (81 use reports) and eating (60 use reports). This paper contains an overview of these use reports, but the focus is on the use for herbal tea as it is the one most frequently mentioned.

What do people think of when they are asked about plant species gathered in the wild?

The link between the wild and gardens

Although asked about plant species growing in the wild, almost one third of the plants mentioned grow in people's gardens (Figure 4). One reason is that some of the plant species mentioned were originally gathered in the wild, but are now cultivated in gardens at home (e.g. Rubus idaeus). The respondents think of these species now grown in gardens as wild.

The respondents also seem to link their answers mentally with their uses. By way of example, respondents who mentioned plants collected in the wild for homemade remedies continued to list all the plants they know which are used in homemade remedies, even if they are grown in gardens (e.g. ointment from Hypericum perforatum L. - growing in the wild - was subsequently linked to Calendula officinalis L. which grows in gardens). Other respondents thought of herbal tea plant species (e.g. starting with Alchemilla sp., Thymus serphyllum L. agg. - which grow in the wild - and then followed by Melissa officinalis L. and Matricaria chamomilla L.- which grow in gardens).

\section{The link between gathering and teas}

Plants used for herbal tea were often named first when respondents were asked about plant species gathered in the wild (most use reports in the nutrition category (127 use reports)), within the nutrition category drinking 
Table 2 Plant species gathered in the wild and their uses in the Biosphere Reserve Grosses Walsertal (free lists, $\mathbf{n}=\mathbf{3 6}$, frequency of mentions $>\mathbf{2}$, sorted by frequency $f$ and rank $R$

\begin{tabular}{|c|c|c|c|c|c|c|c|c|}
\hline$f$ & Scientific name & $\begin{array}{l}\text { German name } \\
\text { (local) }\end{array}$ & Family & $\begin{array}{l}\text { Organ } \\
\text { used }\end{array}$ & Use category & Application & Preparation & $\begin{array}{l}\text { MED/VET uses and } \\
\text { efficacy (if VET and } \\
\text { if specific animals } \\
\text { mentioned, animal } \\
\text { in brackets) }\end{array}$ \\
\hline 30 & Alchemilla alpina & Silbermäntele & Rosaceae & $\begin{array}{l}\text { aerial } \\
\text { parts }\end{array}$ & MED & drink & tea & $\begin{array}{l}\text { for women*, } \\
\text { incontinence }\end{array}$ \\
\hline 30 & $\begin{array}{l}\text { Alchemilla } \\
\text { vulgaris L. agg. }\end{array}$ & Frauenmäntele & Rosaceae & $\begin{array}{l}\text { aerial } \\
\text { parts }\end{array}$ & MED & drink & tea & $\begin{array}{l}\text { for women*, } \\
\text { algomenorrhea, } \\
\text { regulative, kidney } \\
\text { and } \\
\text { bladder trouble, } \\
\text { incontinence }\end{array}$ \\
\hline \multirow[t]{6}{*}{29} & $\begin{array}{l}\text { Calendula } \\
\text { officinalis } \mathrm{L} \text {. }\end{array}$ & Ringelblume & Asteraceae & flower & MEDNET & grease & ointment & $\begin{array}{l}\text { wounds, varicose } \\
\text { veins, } \\
\text { afterbirth (cows) }\end{array}$ \\
\hline & & & & & MED & rubbing in & schnapps & \\
\hline & & & & & MED & bathing & tea & veins \\
\hline & & & & & MED/NUT & drink & tea & $\begin{array}{l}\text { blood circulation in } \\
\text { feet, stomach and } \\
\text { intestine, cancer }\end{array}$ \\
\hline & & & & & NUT & food & biscuit & \\
\hline & & & & & DEC & decoration & salad & \\
\hline \multirow[t]{13}{*}{29} & $\begin{array}{l}\text { Sambucus } \\
\text { nigra L. }\end{array}$ & $\begin{array}{l}\text { Holder, } \\
\text { Holunder }\end{array}$ & Caprifoliaceae & flower & MED & application & $\begin{array}{l}\text { heated in linen } \\
\text { bag over steam }\end{array}$ & $\begin{array}{l}\text { bronchial catarrh, } \\
\text { breast inflammation } \\
\text { in nursing mothers }\end{array}$ \\
\hline & & & & & MED/NUT & drink & tea & $\begin{array}{l}\text { calmative, } \\
\text { diaphoretic, } \\
\text { perspiration, cough }\end{array}$ \\
\hline & & & & & NUT & drink & syrup & \\
\hline & & & & & NUT & food & omelette & \\
\hline & & & & & NUT & food & biscuit & \\
\hline & & & & leaf & MED/NUT & drink & tea & $\begin{array}{l}\text { calmative, } \\
\text { diaphoretic, } \\
\text { perspiration, cough }\end{array}$ \\
\hline & & & & fruit & MED/NUT & drink & juice & $\begin{array}{l}\text { strengthening the } \\
\text { body's defences }\end{array}$ \\
\hline & & & & & MED/NUT & food & jam & cold, cough \\
\hline & & & & & MED/NUT & food & dried & diarrhoea \\
\hline & & & & & NUT & drink & wine & \\
\hline & & & & bark & MED & grease & ointment & rheumatism \\
\hline & & & & twig & $\mathrm{OTH}$ & instrument & whistle & \\
\hline & & & & tree & $\mathrm{OTH}$ & planting & n.s. & $\begin{array}{l}\text { protection against } \\
\text { lightning }\end{array}$ \\
\hline \multirow[t]{2}{*}{28} & $\begin{array}{l}\text { Achillea } \\
\text { millefolium agg. }\end{array}$ & Schafgarbe & Asteraceae & $\begin{array}{l}\text { aerial } \\
\text { parts }\end{array}$ & MED & bathing & tea & rheumatism \\
\hline & & & & & MED/NUT & drink & tea & $\begin{array}{l}\text { anti-inflammatory, } \\
\text { for women*, } \\
\text { menopause, } \\
\text { stomach, } \\
\text { circulation, neuritis } \\
\text { and pain, cold, blood } \\
\text { purification, kidney } \\
\text { and bladder trouble }\end{array}$ \\
\hline
\end{tabular}


Table 2 Plant species gathered in the wild and their uses in the Biosphere Reserve Grosses Walsertal (free lists, $\mathbf{n}=36$, frequency of mentions $>\mathbf{2}$, sorted by frequency $f$ and rank $R$ (Continued)

\begin{tabular}{|c|c|c|c|c|c|c|c|c|}
\hline & & & & & NUT & drink & liqueur & \\
\hline & & & & & VET & food & tea & $\begin{array}{l}\text { anti-inflammatory, } \\
\text { antibacterial (bees) }\end{array}$ \\
\hline & & & & leaf & NUT & food & "spinach" & $\begin{array}{l}\text { (together with } \\
\text { Urtica dioica) }\end{array}$ \\
\hline & & & & & NUT & food & salad & \\
\hline \multirow[t]{4}{*}{28} & $\begin{array}{l}\text { Hypericum } \\
\text { perforatum } \mathrm{L} \text {. }\end{array}$ & Johanniskraut & Hyperiaceae & $\begin{array}{l}\text { aerial } \\
\text { parts }\end{array}$ & MED & drink & tea & $\begin{array}{l}\text { diaphoretic, } \\
\text { indigestion, } \\
\text { calmative, } \\
\text { anti-depressive }\end{array}$ \\
\hline & & & & & MED/NET & grease & oil & $\begin{array}{l}\text { abrasion of vertical } \\
\text { column, } \\
\text { inflammation, } \\
\text { abscess, wounds, } \\
\text { erythema, } \\
\text { actinodermatitis, } \\
\text { earache, wounds } \\
\text { (sows); earache (cats) }\end{array}$ \\
\hline & & & & & MED & grease & ointment & \\
\hline & & & & & OTH & fragrance & herb pillow & calmative \\
\hline \multirow[t]{2}{*}{26} & Rhododendron sp. & Alpenrose & Ericaceae & flower & MED & drink & tea & $\begin{array}{l}\text { cough, croakiness, } \\
\text { bronchitis, cold, } \\
\text { blood purifying }\end{array}$ \\
\hline & & & & & NUT & food & jam & \\
\hline \multirow[t]{12}{*}{23} & Urtica dioica L. & Brennnessel & Urticaceae & $\begin{array}{l}\text { aerial } \\
\text { parts }\end{array}$ & MED/NUT & drink & tea & (blood) purifying \\
\hline & & & & & MED/NUT & food & "spinach" & $\begin{array}{l}\text { spring tiredness, } \\
\text { iron deficiency }\end{array}$ \\
\hline & & & & & NUT & food & onion cake & \\
\hline & & & & & NUT & food & herb salt & \\
\hline & & & & & $\mathrm{OTH}$ & washing & tea & hair \\
\hline & & & & & OTH & fertiliser & tea & \\
\hline & & & & & OTH & insecticide & tea & \\
\hline & & & & root & MED & bathing & tea & calmative \\
\hline & & & & & MED & washing & tea & hair \\
\hline & & & & & MED & washing & schnapps & hair \\
\hline & & & & seed & NUT & food & biscuit & \\
\hline & & & & & NUT & food & bread & \\
\hline \multirow[t]{6}{*}{22} & Rubus idaeus L. & Himbeere & Rosaceae & leaf & MED/NUT & drink & tea & $\begin{array}{l}\text { for women*, blood } \\
\text { purifying, diuretic }\end{array}$ \\
\hline & & & & fruit & MED/NUT & drink & liqueur & $\begin{array}{l}\text { circulation, stomach } \\
\text { upset }\end{array}$ \\
\hline & & & & & MED/NUT & drink & schnapps & $\begin{array}{l}\text { stomach and } \\
\text { intestine }\end{array}$ \\
\hline & & & & & NUT & food & raw & \\
\hline & & & & & NUT & food & jam & \\
\hline & & & & & NUT & food & cake & \\
\hline 21 & Mentha sp. & Pfefferminze & Lamiaceae & $\begin{array}{l}\text { aerial } \\
\text { parts }\end{array}$ & MED/NUT & drink & tea & $\begin{array}{l}\text { stimulating, intensive } \\
\text { taste, stomach ache, } \\
\text { cooling, cough }\end{array}$ \\
\hline
\end{tabular}


Table 2 Plant species gathered in the wild and their uses in the Biosphere Reserve Grosses Walsertal (free lists, $\mathbf{n}=\mathbf{3 6}$, frequency of mentions $>\mathbf{2}$, sorted by frequency $f$ and rank $R$ (Continued)

\begin{tabular}{|c|c|c|c|c|c|c|c|c|}
\hline \multirow[t]{5}{*}{20} & $\begin{array}{l}\text { Plantago } \\
\text { lanceolata L. }\end{array}$ & Spitzwegerich & Plantaginaceae & $\begin{array}{l}\text { leaf } \\
\text { (leaf } \\
\text { fibres) }\end{array}$ & MED & application & raw & earache \\
\hline & & & & & MED & application & raw & wounds, insect bite \\
\hline & & & & & MED/NET & rub in & schnapps & $\begin{array}{l}\text { sprain, muscles, } \\
\text { rheumatism, venous } \\
\text { turgor, joints, } \\
\text { back (cows) }\end{array}$ \\
\hline & & & & & MED/NUT & drink & syrup & cough, lung \\
\hline & & & & & NUT & drink & tea & \\
\hline \multirow[t]{2}{*}{20} & Arnica montana L. & Arnika & Asteraceae & flower & MED & grease & oil & \\
\hline & & & & & MED & grease & ointment & \\
\hline \multirow[t]{2}{*}{20} & $\begin{array}{l}\text { Primula veris } \\
\text { L/Primula }\end{array}$ & $\begin{array}{l}\text { Schlüsselblume, } \\
\text { Himmelschlüssel }\end{array}$ & Primulaceae & flower & MED & drink & tea & $\begin{array}{l}\text { calmative, diuretic, } \\
\text { cough }\end{array}$ \\
\hline & & & & & MED/NUT & food & syrup & cough \\
\hline \multirow[t]{6}{*}{19} & Abies alba & Tanne & Pinaceae & twig & MED & grease & ointment & blistering ointment \\
\hline & & & & & MED & grease & heated & $\begin{array}{l}\text { blistering ointment, } \\
\text { drawing salve }\end{array}$ \\
\hline & & & & & DEC & decoration & $\begin{array}{l}\text { decoration of } \\
\text { herder's } \\
\text { hat and cattle for } \\
\text { "Alpabtrieb" }\end{array}$ & \\
\hline & & & & leaf & NUT & drink & tea & \\
\hline & & & & resin & MED/NUT & drink & schnapps & allergic coryza \\
\hline & & & & cone & MED & drink & schnapps & allergic coryza \\
\hline \multirow[t]{3}{*}{19} & $\begin{array}{l}\text { Matricaria } \\
\text { chamomilla L. }\end{array}$ & $\begin{array}{l}\text { Kamille, } \\
\text { Opflblümli }\end{array}$ & Asteraceae & flower & $\begin{array}{l}\text { MED/NUT/ } \\
\text { VET }\end{array}$ & drink & tea & $\begin{array}{l}\text { stomach ache, } \\
\text { flatulence, } \\
\text { diarrhoea (calves) }\end{array}$ \\
\hline & & & & & MED & grease & ointment & drawing salve \\
\hline & & & & & $\mathrm{OTH}$ & fragrance & herb pillow & calmative \\
\hline \multirow[t]{3}{*}{18} & $\begin{array}{l}\text { Thymus } \\
\text { serpyllum }\end{array}$ & $\begin{array}{l}\text { Thymian, } \\
\text { Quendel }\end{array}$ & Lamiaceae & $\begin{array}{l}\text { aerial } \\
\text { parts }\end{array}$ & MED & bathing & tea & cold \\
\hline & & & & & MED/NUT & drink & tea & $\begin{array}{l}\text { cold, diabetes, } \\
\text { blood purification }\end{array}$ \\
\hline & & & & & NUT & food & spice & \\
\hline \multirow[t]{5}{*}{18} & $\begin{array}{l}\text { Salvia } \\
\text { ffficinalis. }\end{array}$ & Salbei & Lamiaceae & leaf & MED & gurgling & tea & sore throat, angina \\
\hline & & & & & MED & food & raw & sore throat \\
\hline & & & & & MED/NUT & drink & tea & stomach, pyelitis \\
\hline & & & & & $\mathrm{OTH}$ & fragrance & herb pillow & calmative \\
\hline & & & & & $\mathrm{OTH}$ & fumigation & incense & \\
\hline \multirow[t]{2}{*}{17} & Trifolium & (Rot)klee & Fabaceae & flower & MED/NUT & drink & tea & cancer \\
\hline & & & & & NUT & food & raw & \\
\hline \multirow[t]{2}{*}{16} & $\begin{array}{l}\text { Taraxacum } \\
\text { officinale agg. }\end{array}$ & $\begin{array}{l}\text { Schwiblume, } \\
\text { Löwenzahn }\end{array}$ & Asteraceae & leaf & NUT & food & salad & (with potatoes) \\
\hline & & & & & NUT & food & "spinach" & (with Urtica dioica) \\
\hline
\end{tabular}


Table 2 Plant species gathered in the wild and their uses in the Biosphere Reserve Grosses Walsertal (free lists, $\mathbf{n}=\mathbf{3 6}$, frequency of mentions $>\mathbf{2}$, sorted by frequency $f$ and rank $R$ (Continued)

\begin{tabular}{|c|c|c|c|c|c|c|c|c|}
\hline & & & & & NUT & food & onion cake & \\
\hline & & & & flower & NUT & food & syrup ("honey”) & \\
\hline & & & & & NUT & drink & syrup & \\
\hline & & & & root & n.s. & n.s. & n.s. & \\
\hline \multirow[t]{4}{*}{16} & Vaccinium myrtillus L. & Heidelbeere & Ericaceae & fruit & $\begin{array}{l}\text { MED/NUT/ } \\
\text { VET }\end{array}$ & food & raw & costiveness, diarrhoea \\
\hline & & & & & $\begin{array}{l}\text { MED/NUT/ } \\
\text { VET }\end{array}$ & food & dried & costiveness, diarrhoea \\
\hline & & & & & $\begin{array}{l}\text { MED/NUT/ } \\
\text { VET }\end{array}$ & food & jam & costiveness, diarrhoea \\
\hline & & & & leaf & NUT & drink & tea & \\
\hline \multirow[t]{6}{*}{15} & $\begin{array}{l}\text { Melissa } \\
\text { officinalis L. }\end{array}$ & Zitronen-Melisse & Lamiaceae & $\begin{array}{l}\text { aerial } \\
\text { parts }\end{array}$ & NUT & drink & syrup & \\
\hline & & & & & NUT & drink & tea & \\
\hline & & & & & $\mathrm{OTH}$ & fragrance & herb pillow & calmative \\
\hline & & & & leaf & NUT & food & omelette & \\
\hline & & & & & NUT & drink & punch & \\
\hline & & & & & DEC & decoration & raw & \\
\hline \multirow[t]{2}{*}{15} & $\begin{array}{l}\text { Fragaria } \\
\text { vesca } \mathrm{L} \text {. }\end{array}$ & Erdbeere & Rosaceae & leaf & MED/NUT & drink & tea & $\begin{array}{l}\text { blood purifying, } \\
\text { diuretic, for women* }\end{array}$ \\
\hline & & & & fruit & NUT & food & raw & \\
\hline \multirow[t]{2}{*}{15} & Malva neglecta & Käspappel & Malvaceae & leaf & MED & application & raw & suppurative spots \\
\hline & & & & & MED/NET & bathing & tea & $\begin{array}{l}\text { suppurative spots, } \\
\text { wounds, healing of } \\
\text { fractures, supports } \\
\text { perfusion, infantile } \\
\text { eczema, } \\
\text { neurodermatitis, } \\
\text { swollen foot (cows) }\end{array}$ \\
\hline \multirow[t]{2}{*}{14} & Tussilago & Huflattich & Asteraceae & leaf & MED & application & raw & wounds \\
\hline & & & & flowers & MED/NUT & drink & tea & cough \\
\hline 14 & $\begin{array}{l}\text { Juniperus communis } \\
\mathrm{L} \text {. }\end{array}$ & $\begin{array}{l}\text { Räckholder, } \\
\text { Wacholder }\end{array}$ & Cupressaceae & leaf & MED/NUT & drink & tea & $\begin{array}{l}\text { ("Alptee" with } \\
\text { Rhododendron sp.), } \\
\text { diuretic }\end{array}$ \\
\hline \multirow[t]{2}{*}{14} & $\begin{array}{l}\text { Equisetum } \\
\text { arvense L. }\end{array}$ & $\begin{array}{l}\text { Acker- } \\
\text { Schachtelhalm }\end{array}$ & Equisetaceae & $\begin{array}{l}\text { aerial } \\
\text { parts }\end{array}$ & MED & bathing & tea & $\begin{array}{l}\text { back pain, articular } \\
\text { gout, kidney and } \\
\text { bladder trouble, } \\
\text { for women* }\end{array}$ \\
\hline & & & & & OTH & fertiliser & tea & (with Urtica dioica) \\
\hline 14 & Tilia sp. & Linde & Tiliaceae & flowers & MED/NUT & drink & tea & $\begin{array}{l}\text { calmative, } \\
\text { diaphoretic, } \\
\text { cough, fever }\end{array}$ \\
\hline 13 & Bellis perennis L. & Gänseblümchen & Asteraceae & flowers & NUT & decoration & salad & \\
\hline \multirow[t]{2}{*}{12} & Rubus fruticosus agg. & Brombeere & Rosaceae & fruit & NUT & food & raw & \\
\hline & & & & & NUT & food & jam & \\
\hline \multirow[t]{3}{*}{11} & Ribes nigrum L. & $\begin{array}{l}\text { Schwarze } \\
\text { Johannisbeere }\end{array}$ & Grossulariaceae & leaf & MED/NUT & drink & schnapps & $\begin{array}{l}\text { stomach and } \\
\text { intestine }\end{array}$ \\
\hline & & & & & MED/NUT & drink & juice & vitamins \\
\hline & & & & & NUT & drink & liqueur & \\
\hline
\end{tabular}


Table 2 Plant species gathered in the wild and their uses in the Biosphere Reserve Grosses Walsertal (free lists, $\mathbf{n}=\mathbf{3 6}$, frequency of mentions $>\mathbf{2}$, sorted by frequency $f$ and rank $R$ (Continued)

\begin{tabular}{|c|c|c|c|c|c|c|c|c|}
\hline & & & & & NUT & drink & tea & \\
\hline & & & & fruit & NUT & food & jam & \\
\hline \multirow[t]{8}{*}{10} & $\begin{array}{l}\text { Peucedanum } \\
\text { ostruthium (L.) }\end{array}$ & Meisterwurz & Apiaceae & root & MED & grease & ointment & $\begin{array}{l}\text { wounds, } \\
\text { anti-inflammatory }\end{array}$ \\
\hline & & & & & MED & chewing & raw or dried & toothache \\
\hline & & & & & MED/NUT & drink & schnapps & circulation \\
\hline & & & & & MED/NET & fumigation & incense & $\begin{array}{l}\text { disinfection, navel } \\
\text { infection of calves }\end{array}$ \\
\hline & & & & & VET & grease & ointment & sick foot (cows) \\
\hline & & & & & OTH & $\begin{array}{l}\text { carrying in } \\
\text { pocket }\end{array}$ & raw & $\begin{array}{l}\text { protection from } \\
\text { disease }\end{array}$ \\
\hline & & & & $\begin{array}{l}\text { root } \\
\text { and/or } \\
\text { leaf }\end{array}$ & MED/NET & bath & tea & wounds, claws \\
\hline & & & & & VET & application & raw (crushed) & $\begin{array}{l}\text { claw disease } \\
\text { ("der Wilder") }\end{array}$ \\
\hline 10 & $\begin{array}{l}\text { Artemisa } \\
\text { absinthium L. }\end{array}$ & Wermut & Asteraceae & n.s. & n.s. & n.s. & n.s. & \\
\hline 9 & $\begin{array}{l}\text { Trifolium } \\
\text { repens L. }\end{array}$ & Weißklee & Fabaceae & flower & MED/NUT & drink & tea & for women* \\
\hline \multirow[t]{3}{*}{8} & $\begin{array}{l}\text { Origanum } \\
\text { vulgare L. }\end{array}$ & Majoran & Lamiaceae & $\begin{array}{l}\text { aerial } \\
\text { parts }\end{array}$ & MED & grease & ointment & cough \\
\hline & & & & & NUT & food & spice & \\
\hline & & & & & NUT & drink & tea & \\
\hline \multirow[t]{2}{*}{8} & Sambucus & Roter Holunder & Caprifoliaceae & fruit & MED/NUT & drink & juice & cold, cough \\
\hline & & & & & MED/NUT & food & jelly & cough \\
\hline \multirow[t]{4}{*}{8} & Pinus mugo & Latsche & Pinaceae & leaf & MED/NUT & food & syrup ("honey") & cold, cough \\
\hline & & & & & NUT & drink & liqueur & \\
\hline & & & & & NUT & drink & tea & \\
\hline & & & & & $\mathrm{OTH}$ & fumigation & incense & \\
\hline \multirow[t]{3}{*}{8} & Euphrasia & Augentrost & Scrophulariaceae & flowers & MED & application & raw (with dew) & eyes \\
\hline & & & & $\begin{array}{l}\text { aerial } \\
\text { parts }\end{array}$ & MED & bathing & tea & ophthalmitis \\
\hline & & & & & NUT & drink & tea & \\
\hline \multirow[t]{5}{*}{8} & $\begin{array}{l}\text { Rosmarinus } \\
\text { officinalis }\end{array}$ & Rosmarin & Lamiaceae & $\begin{array}{l}\text { aerial } \\
\text { parts }\end{array}$ & MED & grease & ointment & $\begin{array}{l}\text { (with Peucedanum } \\
\text { osthrutium) }\end{array}$ \\
\hline & & & & & MED/NUT & drink & tea & stomach \\
\hline & & & & & NUT & drink & liqueur & \\
\hline & & & & & NUT & food & omelette & \\
\hline & & & & & DEC & decoration & $\begin{array}{l}\text { decoration of } \\
\text { herder's hat for } \\
\text { "Alpabtrieb" }\end{array}$ & \\
\hline \multirow[t]{3}{*}{7} & Rosa sp. & Rose & Rosaceae & flower & MED/NUT & drink & tea & for women* \\
\hline & & & & & OTH & grease & ointment & \\
\hline & & & & fruit & NUT & drink & tea & \\
\hline
\end{tabular}


Table 2 Plant species gathered in the wild and their uses in the Biosphere Reserve Grosses Walsertal (free lists, $\mathbf{n}=\mathbf{3 6}$, frequency of mentions $>\mathbf{2}$, sorted by frequency $\mathbf{f}$ and rank $\mathbf{R}$ (Continued)

\begin{tabular}{|c|c|c|c|c|c|c|c|c|}
\hline \multirow[t]{2}{*}{7} & Monarda didyma & Gold-Melisse & Lamiaceae & flower & MED/NUT & drink & tea & heart strengthening \\
\hline & & & & & NUT & drink & syrup & \\
\hline \multirow[t]{6}{*}{7} & Symphytum & Beinwell & Boraginaceae & leaf & MED & application & raw (smashed) & bursitis \\
\hline & & & & & NUT & food & salad & \\
\hline & & & & & NUT & food & omelette & \\
\hline & & & & & NUT & drink & tea & \\
\hline & & & & root & MED/NET & grease & ointment & $\begin{array}{l}\text { bursitis, feet, joints, } \\
\text { violent pressure, } \\
\text { wounds }\end{array}$ \\
\hline & & & & & MED/NET & bathing & tea & wounds \\
\hline \multirow[t]{4}{*}{7} & Lavandula & Lavendel & Lamiaceae & flower & MED & bathing & tea & brain strengthening \\
\hline & & & & & $\mathrm{OTH}$ & fragrance & herb pillow & calmative \\
\hline & & & & & $\mathrm{OTH}$ & hanging up & bunch & moths \\
\hline & & & & & OTH & fumigation & incense & \\
\hline \multirow[t]{4}{*}{6} & Betula pendula & Birke & Betulaceae & leaf & MED & washing & tea & hair \\
\hline & & & & & MED/NUT & drink & tea & for women* \\
\hline & & & & & NUT & food & salad & \\
\hline & & & & juice & MED/NUT & drink & raw & spring cure \\
\hline \multirow[t]{3}{*}{6} & $\begin{array}{l}\text { Verbascum } \\
\text { densiflorum } \\
\text { BERTOL. }\end{array}$ & Königskerze & Scorphulariaceae & flowers & MED & grease & ointment & $\begin{array}{l}\text { otitis media, } \\
\text { bronchitis, } \\
\text { mucous obstruction }\end{array}$ \\
\hline & & & & & MED & drink & tincture & \\
\hline & & & & & NUT & drink & tea & \\
\hline \multirow[t]{3}{*}{6} & Potentilla & Blutwurz & Rosaceae & root & MED & application & raw & haemostatic \\
\hline & & & & & MED/NUT & drink & schnapps & stomach \\
\hline & & & & & $\begin{array}{l}\text { MED/NUT/ } \\
\text { VET }\end{array}$ & drink & tea & stomach, diarrhoea \\
\hline \multirow[t]{3}{*}{6} & Allium & Bärlauch & Alliaceae & leaf & NUT & food & soup & \\
\hline & & & & & NUT & food & "spinach" & \\
\hline & & & & & NUT & food & "pesto" & \\
\hline 5 & $\begin{array}{l}\text { Lotus } \\
\text { corniculatus L. }\end{array}$ & Hornklee & Fabaceae & flower & NUT & drink & tea & \\
\hline \multirow[t]{3}{*}{5} & $\begin{array}{l}\text { Glechoma hederaceae } \\
\mathrm{L} \text {. }\end{array}$ & Gundelrebe & Lamiaceae & $\begin{array}{l}\text { aerial } \\
\text { parts }\end{array}$ & NUT & food & soup & \\
\hline & & & & & NUT & food & salad & \\
\hline & & & & & NUT & food & spread & \\
\hline 4 & $\begin{array}{l}\text { Anthyllis } \\
\text { vulneraria L. }\end{array}$ & Wundklee & Fabaceae & flower & NUT & drink & tea & \\
\hline 4 & $\begin{array}{l}\text { Chenopodium } \\
\text { bonus-henricus L. }\end{array}$ & Guter Heinrich & Chenopodiaceae & leaf & NUT & food & "spinach" & \\
\hline 4 & $\begin{array}{l}\text { Vaccinium } \\
\text { vitis-idaeae L. }\end{array}$ & Preiselbeere & Ericaceae & fruit & NUT & food & jam & \\
\hline
\end{tabular}


Table 2 Plant species gathered in the wild and their uses in the Biosphere Reserve Grosses Walsertal (free lists, $\mathbf{n}=\mathbf{3 6}$, frequency of mentions $>\mathbf{2}$, sorted by frequency $\mathbf{f}$ and $\operatorname{rank} \mathbf{R}$ (Continued)

\begin{tabular}{|c|c|c|c|c|c|c|c|c|}
\hline 4 & $\begin{array}{l}\text { Gentiana } \\
\text { lutea L. }\end{array}$ & Gelber Enzian & Gentianaceae & root & MED/NUT & drink & schnapps & $\begin{array}{l}\text { stomach and } \\
\text { intestine }\end{array}$ \\
\hline 4 & $\begin{array}{l}\text { Valeriana } \\
\text { officinalis agg. }\end{array}$ & Baldrian & Valerianaceae & root & MED & drink & tincture & cardiotonic \\
\hline \multirow[t]{2}{*}{4} & Sorbus & Eberesche & Rosaceae & fruit & NUT & drink & schnapps & \\
\hline & & & & & NUT & food & jam & \\
\hline \multirow[t]{2}{*}{4} & $\begin{array}{l}\text { Galium } \\
\text { odoratum (L.) } \\
\text { SCOP. }\end{array}$ & $\begin{array}{l}\text { Echter } \\
\text { Waldmeister }\end{array}$ & Rubiaceae & $\begin{array}{l}\text { aerial } \\
\text { parts }\end{array}$ & MED/NUT & drink & tea & $\begin{array}{l}\text { for women*, } \\
\text { anti-depressive }\end{array}$ \\
\hline & & & & & NUT & drink & punch & \\
\hline 4 & $\begin{array}{l}\text { Plantago } \\
\text { major L. }\end{array}$ & Breitwegerich & Plantaginaceae & $\begin{array}{l}\text { leaf } \\
\text { (leaf } \\
\text { fibres) }\end{array}$ & MED & application & raw & earache \\
\hline \multirow[t]{4}{*}{4} & Tropaeolum majus & Kapuzinerkresse & Tropaeolaceae & flower & NUT & food & herb salt & \\
\hline & & & & & NUT & food & salad & \\
\hline & & & & leaf & NUT & food & salad & \\
\hline & & & & & VET & food & raw & immune system \\
\hline 3 & Carum carvi L. & Kümmel & Apiaceae & seed & MED/NUT & drink & tea & stomach, flatulence \\
\hline 3 & $\begin{array}{l}\text { Verbena } \\
\text { officinalis L. }\end{array}$ & Eisenkraut & Verbeneceae & $\begin{array}{l}\text { aerial } \\
\text { parts }\end{array}$ & MED & drink & tea & wounds \\
\hline \multirow[t]{2}{*}{3} & $\begin{array}{l}\text { Levisticum officinale } \\
\text { W.D.J.KOCH }\end{array}$ & Liebstöckl & Apiaceae & $\begin{array}{l}\text { aerial } \\
\text { parts }\end{array}$ & NUT & food & spice & \\
\hline & & & & & MEDNET & bathing & tea & $\begin{array}{l}\text { open wounds, } \\
\text { purulent wounds }\end{array}$ \\
\hline \multirow[t]{3}{*}{3} & $\begin{array}{l}\text { Geranium } \\
\text { robertianum L. }\end{array}$ & Storchschnabel & Geraniaceae & $\begin{array}{l}\text { aerial } \\
\text { parts }\end{array}$ & MED & drink & tea & $\begin{array}{l}\text { women to become } \\
\text { pregnant }\end{array}$ \\
\hline & & & & & VET & food & raw & $\begin{array}{l}\text { animals to become } \\
\text { pregnant }\end{array}$ \\
\hline & & & & flower & $\mathrm{OTH}$ & grease & ointment & \\
\hline 3 & $\begin{array}{l}\text { Centaurea } \\
\text { cyanus L. }\end{array}$ & Kornblume & Asteraceae & flower & NUT & drink & tea & \\
\hline 3 & $\begin{array}{l}\text { Primula } \\
\text { elatior (L.) L. }\end{array}$ & $\begin{array}{l}\text { Wald } \\
\text { Schlüsselblume }\end{array}$ & Primulaceae & flower & NUT & drink & tea & \\
\hline 3 & $\begin{array}{l}\text { Malus domestica } \\
\text { BORKH. }\end{array}$ & Apfel & Rosaceae & $\begin{array}{l}\text { pod of } \\
\text { the fruit }\end{array}$ & NUT & drink & tea & \\
\hline \multirow[t]{2}{*}{3} & Viola tricolor agg. & Veilchen & Violoceae & flower & NUT & drink & punch & \\
\hline & & & & & OTH & grease & ointment & lips, hands \\
\hline \multirow[t]{2}{*}{3} & $\begin{array}{l}\text { Allium } \\
\text { schoenophrasum } \\
\text { subsp. Alpinum L. }\end{array}$ & $\begin{array}{l}\text { Wilder } \\
\text { Schnittlauch }\end{array}$ & Alliaceae & leaf & NUT & food & raw & \\
\hline & & & & & NUT & food & omelette & \\
\hline \multirow[t]{2}{*}{3} & $\begin{array}{l}\text { Humulus } \\
\text { lupulus L. }\end{array}$ & Hopfen & Cannabaceae & fruit & MED/NUT & drink & tea & for women*, cold \\
\hline & & & & & OTH & fragrance & herb pillow & calmative \\
\hline 3 & $\begin{array}{l}\text { Satureja } \\
\text { hortensis L. }\end{array}$ & Bohnenkraut & Lamiaceae & $\begin{array}{l}\text { aerial } \\
\text { parts }\end{array}$ & NUT & food & spice & \\
\hline
\end{tabular}


Table 2 Plant species gathered in the wild and their uses in the Biosphere Reserve Grosses Walsertal (free lists, $\mathbf{n}=\mathbf{3 6}$, frequency of mentions $>\mathbf{2}$, sorted by frequency $\mathbf{f}$ and rank $\mathbf{R}$ (Continued)

\begin{tabular}{|c|c|c|c|c|c|c|c|c|}
\hline 3 & $\begin{array}{l}\text { Petroselinum } \\
\text { crispum (MILL.) FUSS }\end{array}$ & Petersilie & Apiaceae & $\begin{array}{l}\text { aerial } \\
\text { parts }\end{array}$ & MED/NUT & drink & wine & $\begin{array}{l}\text { cardiotonic, } \\
\text { circulation }\end{array}$ \\
\hline 3 & Cetraria islandica & Isländisch Moos & & $\begin{array}{l}\text { aerial } \\
\text { parts }\end{array}$ & MED & drink & syrup & $\begin{array}{l}\text { added to syrup of } \\
\text { Plantago lanceolata }\end{array}$ \\
\hline \multirow[t]{3}{*}{3} & Pimpinella & Bibernell & Apiaceae & root & MED & application & raw (sliced) & between teeth \\
\hline & & & & & MED/NET & bathing & tea & $\begin{array}{l}\text { muscles, wounds; } \\
\text { claws }\end{array}$ \\
\hline & & & & flower & NUT & drink & tea & \\
\hline 3 & Rosa canina agg. & Hagebutte & Rosaceae & fruit & NUT & drink & tea & \\
\hline \multirow[t]{2}{*}{3} & Sedum sp. & Fette Henne & Crassulaceae & leaf & NUT & food & salad & \\
\hline & & & & & NUT & food & cooked & \\
\hline 3 & $\begin{array}{l}\text { Tanacetum } \\
\text { vulgare } \mathrm{L} .\end{array}$ & Rainfarn & Asteraceae & $\begin{array}{l}\text { aerial } \\
\text { parts }\end{array}$ & VET & fumigation & incense & $\begin{array}{l}\text { disinfection } \\
\text { (bee-house) }\end{array}$ \\
\hline \multirow[t]{2}{*}{3} & $\begin{array}{l}\text { Capsella } \\
\text { bursa-pastoris (L.) } \\
\text { MEDIK. }\end{array}$ & Hirtentäschel & Brassicaceae & $\begin{array}{l}\text { aerial } \\
\text { parts }\end{array}$ & MED & grease & tincture & $\begin{array}{l}\text { fracture, inguinal } \\
\text { hernia, tissue } \\
\text { strengthening }\end{array}$ \\
\hline & & & & & NUT & drink & tea & \\
\hline 3 & Viscum album L. & Mistel & Loranthaceae & $\begin{array}{l}\text { aerial } \\
\text { parts }\end{array}$ & MED/NUT & drink & tea & $\begin{array}{l}\text { regulating blood } \\
\text { pressure }\end{array}$ \\
\hline
\end{tabular}

In total, the 892 mentions refer to 140 plant species.) $M E D=$ medicinal, NUT= nutritional, $V E T=$ veterinary, $D E C=$ decoration, $O T H=$ others. $n . s .=$ no statement. Table 2: Plant species gathered in the wild and their uses in the Biosphere Reserve Großes Walsertal (free lists, $n=36$, frequency of mentions $>2$, sorted by frequency $f$ and rank R. In total, the 892 mentions refer to 140 plant species.) $M E D=$ medicinal, $N U T=$ nutritional, $V E T=$ veterinary, $D E C=$ decoration, $O T H=$ others. n.s. $=$ no statement.

* Respondents often generally said that a plant "is good for women". This refers to issues such as algomenorrhea, regulation of menstruation, pregnancy and menopause.

applications (81 use reports), and within this drinking application tea (71 use reports). One reason for this might be local understanding of the term "tea", expressed in the local turn of phrase in the Walser dialect "Gö $m r$ gi te sammla" which is literally translated as "Let's go and collect tea"). This phrase expresses the idea in the study area that gathering plants means gathering plants for herbal tea.

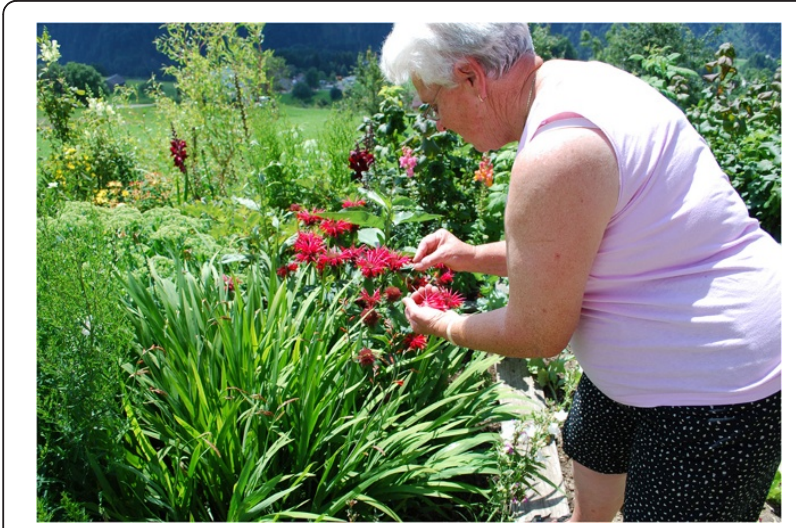

Figure 4 Woman picking Monarda dydima in her garden for tea (Photo: Susanne Grasser).
Bergtee - a community project

Herbal tea has great relevance in the Grosses Walsertal region.

The Bergtee (mountain tea) association was founded in 2003 by a young man and two middle-aged women with the idea of bringing together women in the region who are interested in and knowledgeable about gathering plants for herbal tea. The initiative came from the recognition that they have "something valuable" in their valley. They want to share and exchange the "ancient knowledge" held by women in the valley. At the same time, members of this association are expected to be open to "new" ideas: e.g. nowadays younger members of this association look up recipes in magazines or on the internet. In addition to knowledge passed on orally, when asked for sources of their knowledge, respondents showed popular herb books and excerpts from newspaper articles (the local newspaper has a "herbal knowledge" column) or talked about courses they had attended. "Everything that works counts". The members of the association accept knowledge as something that is dynamic.

When the above-mentioned young man in his early twenties went to Vienna to study, he took some tea from the valley with him. Drinking this tea, he remembered his time as a little boy when he spent several summers 
on an alpine pasture in Grosses Walsertal. The elderly farming women there always sent him to collect "Silbermänetli" (Alchemilla alpina) or other herbal plants to take home to his mother at the end of the summer. The women believed that this gathering activity helped against home sickness. This gathering of herbal plants and his childhood memories somehow bound him to his valley. He then shared the herbal mountain tea with his fellow students in the city as an adult and got the idea of marketing it in the city's tea house where tea from all over the world is sold - but not at that point from Grosses Walsertal. He wanted to share this special and valuable product and so called his mother in the valley to ask if it was possible to send more tea, arrange for women to gather herbal plants and mix blends of Bergtee. This finally led to the creation of the Bergtee community project.

As well as social events (tea afternoons for exchanging knowledge), the project leaders sell herbal tea in local shops, a few local restaurants and selected teahouses. They guarantee high-quality products made from handpicked herbs from the mountain meadows and alpine pastures which are then air dried and carefully processed. They are not interested in producing large quantities, but rather in understanding and valuing the herbal plants and their effects. They want to pass on this understanding, the way they appreciate and value nature and what it offers their customers (see also: [43]).

The project leaders say their ancestors passed on knowledge about plants from generation to generation and that they knew all about the relevance of the position of the sun, phases of the moon, times for gathering, habitats and air quality. In the Bergtee project the women are therefore trying to cultivate this knowledge, pass it on and take it into account when they gather herbal plants for tea.

The project leaders, as well as the women involved who gather plants for the Bergtee project, emphasise attentiveness to nature and appreciation of the treasures it provides. Therefore informal guidelines for gathering (and processing) plants have been established and are highlighted every year in an annual meeting at the start of the gathering season. These guidelines are based on experience over the years and are passed on orally. They are like an informal code on good common practice. The project leaders do not control compliance but have trust in the women who gather herbal plants for the Bergtee association.

The rules of these informal guidelines start with the beginning of the season. The first flowers that appear (e.g. Tussilago farfara L.) are to be left for the bees. When plants are picked, they not all the plants growing at one site should be taken. Where just a few individual plants of a certain species are growing, they should not be picked. Pickers may not walk in the high meadows while gathering (otherwise this will present difficulties for farmers when mowing).

According to their informal guidelines, the best time of day for collecting plants (especially for flowers, e.g. Calendula officinalis L., Hypericum perforatum L., Trifolium pratense L.) is around midday when the sun is shining and at its strongest (as the plants themselves are at their peak then as well). Plants should also be allowed two or three days in the sun to "top up" their energy. With both flowers and leaves, it is important only to take dry ones. The morning dew needs to have dried.

Leaves (e.g. Plantago lanceolata L., Alchemilla vulgaris L. agg., Fragaria vesca L.) have to be inspected carefully. Only healthy ones without holes or any sign of pests or disease may be taken. They have to be gathered while they are still young and fresh, e.g. raspberries (Rubus idaeus L.) and blackberries (Rubus fruticosus L. agg.) are taken before stems become spiky. The leaves of blackcurrants (Ribes nigrum L.) should be picked before the fruits ripen.

Roses (Rosa canina L.) are taken from gardens and only from plants where pesticides have not been used. Only fresh petals may be picked, not from flowers that have almost withered. Any plant species used for herbal tea has to be gathered solely on unfertilised meadows. A frequent statement - also used for advertising the Bergtee - is that "tea herbs should not have heard a car", meaning that plants are not picked near roads, but higher up in the mountains or on the alpine pastures.

Based on experience, the guidelines state that the constellation of stars plays a role in the quality of the herbal plants and in processing and storing them. Some women have good experiences of gathering under specific zodiac signs, but many women also admit that it is not always possible to bear astrology in mind. These women state that plants have to be picked when the weather is right and they have time. One area of knowledge that has been passed on and is recommended by the project leaders is to pick elder flowers (Sambucus nigra L.) at around the full moon. The women find that the flowers retain their yellow colour more, instead of becoming brownish.

They also advise the use of paper or linen bags for gathering. Plants would "sweat" in plastic bags, diminishing their quality. Also for drying purposes, the plants should be well spread out on a white cloth in the dark. No direct sunshine and a bit of a draught allow them to dry evenly. They should be turned every day until they are fully dried which can be recognised by a "crunchy sound" when they are crushed in your hand. The plants are then stored in paper bags which are kept open.

The Bergtee project leaders then collect all the gathered plants from several women in the valley to mix different blends for selling (Figure 5). They always combine 


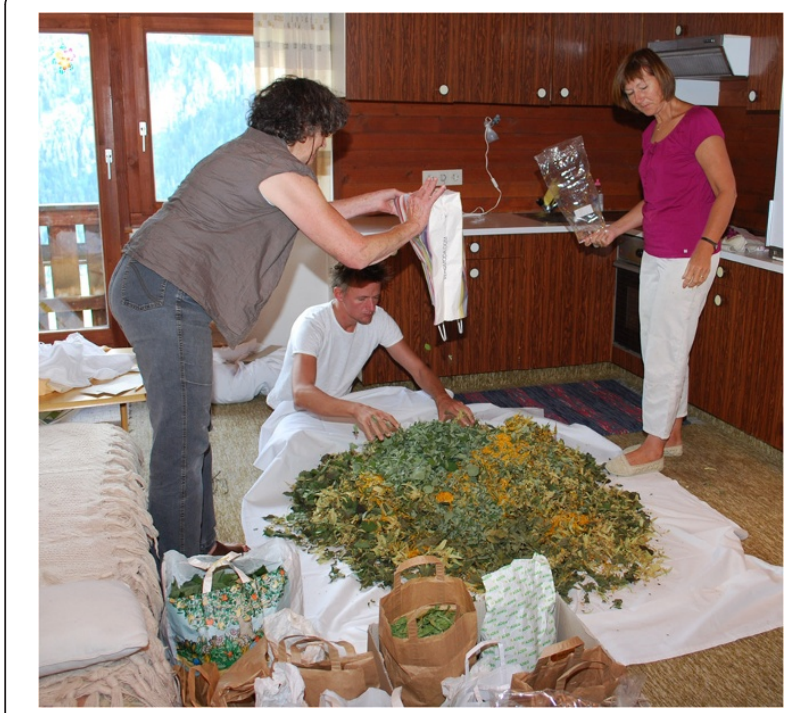

Figure 5 Bergtee project leaders mixing tea herbs (Photo: Susanne Grasser).

seven plant species as there is a traditional saying that no illness will come near when you drink a seven-plant blend. However, the project leaders emphasise that under Austrian law they are not allowed to declare their blends to have any medicinal benefits. Sayings are allowed, e.g. "Grandmother said ribwort is good for coughs" but nothing more than this. Many women recommend following their own intuition when picking plants: if someone is suffering from an illness, the "right" plant to heal this illness will be growing around them (e.g. plenty of flowers of Tussilago farfara L. will be found when someone is suffering from a cough). Attention should be paid to instinct: the plant you feel attracted to will also be the one that is right for your needs at the time. In any case, the women mix the herbal plants intuitively to a well-balanced and tasty blend which is never the same twice. Aesthetics are also important, therefore every blend also comprises some colourful flowers (e.g. Calendula officinalis L., Monarda didyma, Primula veris L.) to appeal to the eye as well.

When the plants are packed (Figure 6) - usually in 30 gram portions - attention is again paid "to the current zodiac sign". Information about which sign is on which day and between which times is looked up in the "Vorarlberger Schreibkalender" [63] or any similar "moon calendar". So called "air signs" (Aquarius, Gemini, Libra) are thought to be best for packing, so that static does not make the dried plants stick to the plastic. For selling purposes, transparent plastic bags are chosen so that people can see what the product looks like.

Usually, about 40 to 45 kilograms of dried plants per year are gathered for the Bergtee association and sold in local shops, a few restaurants and selected tea houses. The women could easily sell more, but say that they just want to gather for as long as they enjoy it. The financial aspect is not a priority. If the packs of tea are sold out, they are sold out and customers have to wait until the harvesting season starts again. Therefore the women of the Bergtee project expect that consumers also experience another relationship with and understanding of the cycle of nature. Furthermore, the plant species are all of equal value (financially as well), i.e. the project leaders pay their pickers the same price per kilo for Urtica dioica L. (growing in large amounts and called a "weed") as for the tiny petals of Monarda didyma.

According to the women, everything in the process from gathering and drying to packing and storing - has to be done with great diligence. The act of gathering plants always has to be done with pleasure otherwise it is said that the pickers' own stress and bad energy will flow into the product. Anyone drinking this tea would also drink in the pickers' stress and anger.

The women emphasise that herbal mountain tea is a product to be enjoyed. When tea is drunk, it provides for a few moments of relaxation. Just a small portion of herbs is enough for a litre of boiled water brewed for ten minutes. Tea should be something sociable - drinking it with other people and using the time for a chat. The Bergtee project also brings women together to exchange their experiences as they are always open to learning something old and new. One woman explained that through the Bergtee project, she was inspired to start gathering again as she knew about it from her parents but never really practiced it herself until then. Now she greatly enjoys this activity and wants to pass on her

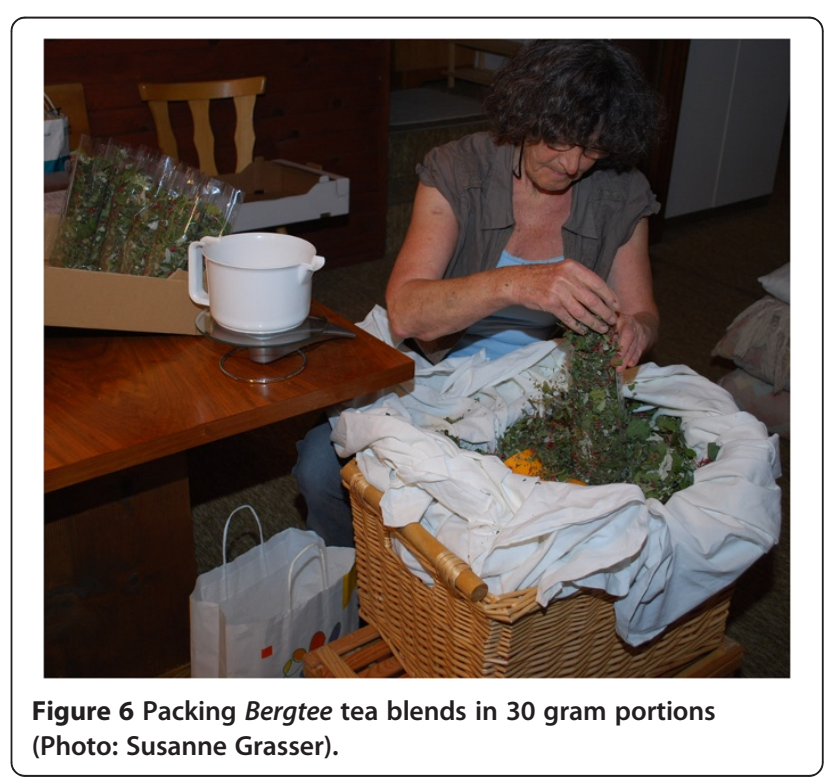


knowledge to her own children who like joining her in the alpine pastures. This reflects regional identity and an awareness of the value of surrounding nature. Therefore Bergtee is more: "Es ist kein Produkt - es ist eine Geschichte" ("It's not a product - it's a history", literally translated [43]).

\section{Alchemilla - a women's project}

Alchemilla is the name of an association of women in the Biosphere Reserve Grosses Walsertal who formed a group in 2006 to deal with the broad issue of herbal plants. Their objective is to show the diversity and value of wild and cultivated plants in the biosphere reserve, pass on their knowledge and traditions and sell products made from the region's plants. For example, they give courses in balsam or soap making, organise herbal walks and ritual ceremonies and offer a diverse programme. Alchemilla is a project by women for women with an economic, ecological and social dimension.

As the focus of this paper is primarily on herbal tea, this project has not been explored in detail. Nevertheless, these Alchemilla women are an important catalyst for developing awareness of the value of plant gathering and usage. Their products and approach are rather "new", introducing various customs and products that are new to the region, while the Bergtee association is geared more towards local traditions. As the Bergtee was founded first, it might also be seen as steering the development of gathering in the region. In any case, both projects share an appreciation of nature and a respectful exposure to natural resources.

\section{Gathering wild plants - revitalising tradition?}

Respondents explained that in the past gathering plants in the wild was a necessity due to poverty. Nature offered food supplements such as dandelion (Taraxacum officinalis agg.) added to potato salad or Good King Henry/poor man's asparagus (Chenopodium bonushenricus L.) and stinging nettle (Urtica dioica L.) which were used as "spinach". Traditional tea blends from the past were also mentioned by respondents. The typical Alptee ("alpine tea"), a mixture of lady's mantle (Alchemilla alpina), juniper (Juniperus communis L.) and Alpine rose (Rhododendron sp.), was gathered and drunk on the alpine pastures where many people from the valley spent the summer months with their cattle. These are plants growing anyway which would otherwise not have been affordable. People relied on self-supply for nutrition and medical care. Gathering plants for eating and drinking was as common as making homemade remedies from several plant species (e.g. ointments from Calendula officinalis L., oil from Hypericum perforatum L., syrup from Abies alba MILL. and Picea abies (L.) H. KARST., bathing with Malva neglecta WALLR. and fumigating using Peucedanum ostruthium (L.) W.D.J. $\mathrm{KOCH})$.

As people in Grosses Walsertal slowly recovered from the Second World War and products became available in the shops, respondents said they were almost proud then of being able to afford to buy items such as spices like caraway (Carum carvi L.) or instant tea bags. Gathering plants was associated with poverty and this was something from which they wanted to be liberated.

It took years - up to the 1970s - before the value of something people gathered and made themselves was appreciated again. The quality of the products themselves became more highly thought of than that of bought products. For one Bergtee association member, this perception was enhanced while she was bringing up her children. She could then look back on her own childhood differently. A shift towards connectedness with nature took place.

Now for many of the respondents, the main purpose of collecting plants is simply the pleasure and satisfaction they find in doing it (Figure 7). They enjoy being

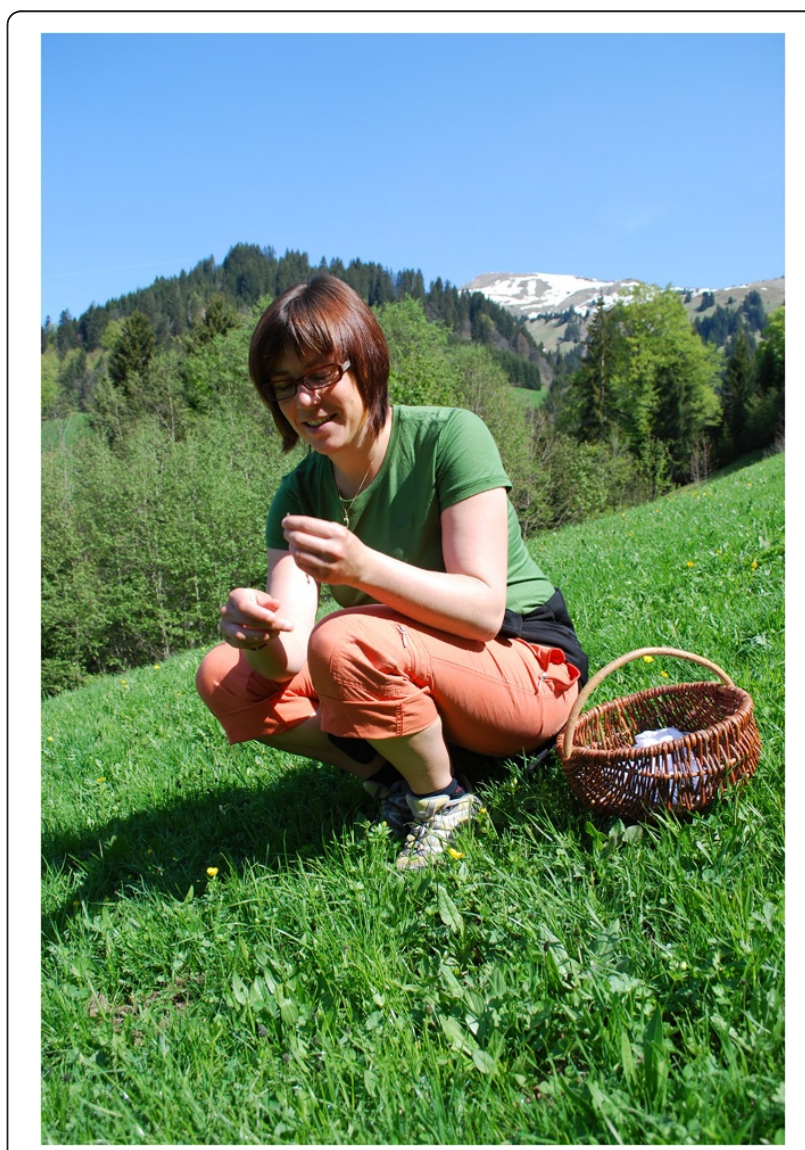

Figure 7 Woman picking tea herbs (e.g. Alchemilla vulgaris, Taraxacum officinale, Plantago lanceolata) (Photo: Susanne Grasser). 
outdoors, experiencing it as a kind of "time off" in a place where they come to rest and benefit from the calmness of nature while still "doing something valuable".

Gathering plants also offers an opportunity for a "social event". Women come together not just to chat, but to "do something useful" (as respondents put it themselves), such as picking berries for example. The respondents would rather meet for a trip to the alpine pastures when it is connected with gathering tea herbs than just go for a walk.

Drinking a cup of tea from the alpine pastures brings back good memories. It allows them to drink in the atmosphere from the past, childhood and beautiful mountains - in this case with good associations rather than still thinking of poverty. There is a certain romance to it. At least for tourists to whom the little tea bags are sold, the product brings with it a sense of holidaying in the beautiful Grosses Walsertal mountains.

Reasons for gathering wild plants have therefore changed over time from being something of necessity to allowing a connectedness with nature, enjoyment and pleasure, with socio-economic factors influencing this activity considerably. Therefore, gathering herbal plants for tea - such as by the two women and young man who founded the Bergtee project - can be perceived as revitalising tradition.

\section{Discussion}

Plants gathered in Grosses Walsertal are comparable to those that appear in other European studies. Those most similar are the free-listed plant species from Eastern Tyrol, Austria [17], where both vegetation and culture are comparable. Nevertheless, the order (frequency, rank and Smith's salience) differs. Some plant species are mentioned noticeably less frequently in Grosses Walsertal than in Eastern Tyrol, e.g. Vaccinium vitis-idaea L. (mentioned by $81 \%$ of respondents in Eastern Tyrol) occurs in lists supplied by Walser people just four times (11\%), Cetraria islandica (mentioned by $44 \%$ of respondents in Eastern Tyrol) just three times (8\%). These plants are less widespread in the alpine regions of Grosses Walsertal. This result supports the proposition that the more common a plant species is in an area, the greater the probability of its popular use [64].

Results from Italy [65] regarding the plant varieties gathered are also comparable to those gathered in Grosses Walsertal, but the importance of each single plant (e.g. expressed through frequency of mentions) differs between the two regions. While Alchemilla millefolium agg., Arnica montana L., Malva neglecta WALLR., Rubus idaeus L., Taraxacum officinalis agg., Urtica dioica L. and Vaccinium myrtillus L. are among the most frequently mentioned plant species in Italy and in the Austrian Grosses Walsertal, Alchemilla vulgaris L. agg.,
Hypericum perforatum L., Sambucus nigra L., Tussilago farfara L. - included in the most frequently mentioned plant species in the Austrian Grosses Walsertal - are taxa quoted in the Italian study by fewer than $10 \%$ of respondents [65]. On the other hand, Tanacetum vulgare L.- among the taxa quoted by at least $40 \%$ of respondents in Italy - is mentioned by just three of the Austrian respondents (8\%). Peucedanum ostruthium (L.) W. D.J.KOCH for veterinary folk medicine - among the taxa quoted by more than $40 \%$ of respondents in Italy has a lower value in the Austrian Grosses Walsertal, quoted by just $26 \%$ of respondents. Fragaria vesca L., Juniperus communis L., Plantago lanceolata L. and Thymus sp. are quoted more often in Grosses Walsertal; Vaccinium vitis-idaea L. and Viola odorata L. are quoted more often in Italy. Indeed, there are other plants that do not occur in both lists.

Plant species also used in Bulgaria [66] include Urtica dioica L., Rosa sp. Rubus idaeus L., Matricaria chamomilla L. and Thymus sp., with many other plants similar to the presented results. In the Mediterranean region, plants such as Thymus sp., Sambucus nigra L., Mentha sp., Melissa officinalis L., Taraxacum officinalis agg., Urtica dioica L. [26,27,67] are similar to those mentioned in Grosses Walsertal.

It is striking that people in Grosses Walsertal do not mention mushrooms which are however mentioned as a source of wild edible plants in other studies on gathering $[7,18]$. They do occur in the region and in participatory observation were also detected as being gathered, but seem to have no "history of gathering" in the valley. The mention of Alchemilla alpina and Alchemilla vulgaris L. agg. at the top of the list in Grosses Walsertal is noteworthy as they are found in just a few studies elsewhere $[12,17,65,68-70]$ (Poland until the $19^{\text {th }}$ century [20]). Respondents refer to the "traditional alpine tea" when they mention Alchemilla sp. Alpine pasturing was a traditional method of livestock management and, to a certain extent, is still practiced in the valley. Gathering plants therefore also reflects people's ways of life: they gather near to where they live [7].

In Grosses Walsertal, the families with the most plant species mentioned are Lamiaceae, Asteraceae and Rosaceae. This is almost similar to studies conducted for example in Spain (Asteraceae, Lamiaceae, Fabaceae, Rosaceae [7,35,71] and Palestine [72]. "Té" in Spain refers to 70 different plant species, the most frequent families being Asteracea and Lamiaceae [67].

As in Grosses Walsertal, the most common use reports elsewhere are as medicine and in food preparation (e.g. Eastern Tyrol, Austria: [17], Bulgaria: [66], Spain: [7]). Plants falling along the food-medicine continuum [73] take up a large part of the species list $[3,4,19]$. Most of the non-crop edible plants and those 
consumed in drinks have medicinal usage (Spain: 77.3\% in [7]). Most frequently applied remedies are teas made from leaves or flowers [17]. In Spain tea (té) is also not primarily associated with Camellia sinensis, but rather with herbal tea plants [67] - the same as in Grosses Walsertal, where people even use the term "collecting tea" instead of "collecting herbal plants".

Even just by looking at the titles of papers by other authors, it is obvious that the focus on ethnobotanical knowledge in Europe is on the alimentary and medicinal use of plants. Other use categories are far less represented, e.g. use reports for dyeing [74] or in the "domestic, ludic, agropastoral, magic/medicinal, religious, handicraft or magic/ritual/propitiatory" categories [25]. Similarly, fewer use reports in these categories were given in Grosses Walsertal, summarised here as "others" - e.g. the "Pfannenfrusi", a little bunch of dried twigs of Calluna vulgaris, which was used in the past to clean pans. Plants gathered for firewood and materials for tools and shelters did not appear in the free lists in Grosses Walsertal. As clearly stated, in this region the idea of gathering is related more to herbal teas than to the scientific concept of wild versus cultivated. It seems that this is the reason why plants used for firewood etc. were not mentioned, since these technological aspects are far removed from local people's associations with the term "plant gathering".

Compared with "earlier literature" [30,49-51] the interviews did not reveal any totally unknown or surprising results. There seems to be no special "Walser knowledge" that is known and practiced only by people in Grosses Walsertal, but rather common plant knowledge spread over the region.

The fact that respondents also listed plants they cultivated in their gardens has also occurred in other studies, e.g. Spain: [7,9,75], Bulgaria [66] and Austria [17]. Christanell [17] argues that this can be explained by a reflection of the history of plant management in the area. Plants which are no longer easily available have been moved slowly into nearby habitats and become cultivated in people's gardens [17]. This may also be true for Grosses Walsertal. One respondent explicitly stated that she dug up a root of Peucedanum ostruthium (L.) W.D.J. $\mathrm{KOCH}$ from the Alpine pasture and planted it in her own garden because she was "too old to climb up the mountain anymore". Nevertheless, we believe that the mention of many plant species (almost one third) grown in people's gardens is more a psychological matter in terms of associations made by respondents when they were asked to name plant species gathered in the wild. When people thought of herbal plant species or plants they use for tea blends, they simply ended up listing all the plants they use, no matter where these plants are growing. For their work on plants traditionally gathered in the Basque Country, Mendez-Baceta et al. [9] also decided to include reports of all species that were referred to by informants as "wild" in their concept of the term, independently of considerations concerning their potential management. The local terms mostly include native species growing in their natural habitat, but sometimes also managed or even promoted by planting their seeds. There are also domesticated species that grew in the area, both cultivated and in the wild. So it was impossible to distinguish between spontaneous or sown species. It is not the botanical or scientific concept of "wild" that counts, but rather local people's own perception and what they associate with this term.

In many of the sites investigated, the activity of plant gathering, use and management seems to be predominantly done by women $[17,76]$.

Guidelines for gathering plants are not particularly well documented - at least not in ethnobotanical scientific literature. However it is still important to present these informal guidelines as they also reflect people's attitude towards nature and the utilisation of nature's products. While in other countries (e.g. Bulgaria: [66]) sustainable collecting methods are not practiced, because people (usually working in companies) do not feel connected to the area and so just pick as much as possible, people in Grosses Walsertal use their environment with great respect and gratitude. It is noteworthy just how much respondents emphasised the importance of the sustainable use of natural resources. It is assumed that this is less due to the fact that Grosses Walsertal is a biosphere reserve than to people's regional identity and appreciation of nature (see also: [47]).

In Bulgaria, for instance, the financial aspects of gathering plants are to the fore [66]. In contrast, making an income from gathered and processed plants is not the main motivation of people in Grosses Walsertal. They primarily gather plants for joy. It is an expression of being connected to nature (see also: [17]). This motivation has developed from a negative perception of wild plant consumption in the past - as a symbol of poverty (see also: $[20,75])$ - to a very positive connotation nowadays. In some countries, it is also linked to tourist activities (e.g. Poland: [20] and Spain: [75]) which has allowed for a revitalisation of wild plant gathering and their use in traditional cuisine as an expression of cultural heritage. It can also be used as marketing for the Biosphere Reserve Grosses Walsertal [43]. However, the strengthening of regional identity outwards (e.g. through marketing the Biosphere Reserve) also consolidates the inward identity and awareness of the value of the nature around people. Therefore, wild plant gathering in Grosses Walsertal is not a vanishing knowledge at risk of being lost forever. Instead it is undergoing a process in which traditions are being revitalised. 
Gathering plants is something many people seem to do in Grosses Walsertal. Nevertheless, the response of many people in the interviews was: "I don't know anything special. It's not worth your while asking me about it". This expresses a lack of awareness of the value of their knowledge and practice. The belief however is that the foundation of the Bergtee association has raised awareness and furthered the exchange of knowledge. It is said that "Walser people do not talk very much" (as one respondent explained) - they are not used to talking about what they do. The Bergtee association provides an opportunity for coming together to chat and discuss people's experiences so that they can all learn from one other. The Bergtee association provides an incentive and motivation for gathering plants. However, perhaps it is not so much the gathering activity that has changed through the Bergtee association, but rather the exchange of knowledge and awareness of its value.

\section{Conclusions}

Gathering of wild plant species is a common practice in Grosses Walsertal. While in the past it was seen as a necessity due to poverty, it is now becoming fashionable again as a pleasurable activity. The shift in reasons for gathering, from necessity to connectivity with nature and the appreciation of homemade products, revitalises the gathering activity and use of plant species gathered in the wild. The Bergtee (mountain tea) association is one example in the Biosphere Reserve Grosses Walsertal that shows the inextricable link between nature and culture. The marketing of plants gathered in the wild for herbal tea projects people's regional identity outwards. However, even more important is local people's own connectedness with the nature around them for a sustainable use of natural resources (Figure 8). The exchange of local knowledge and experiences among the women who gather plants for the Bergtee association sustains their customs and passes on this intangible

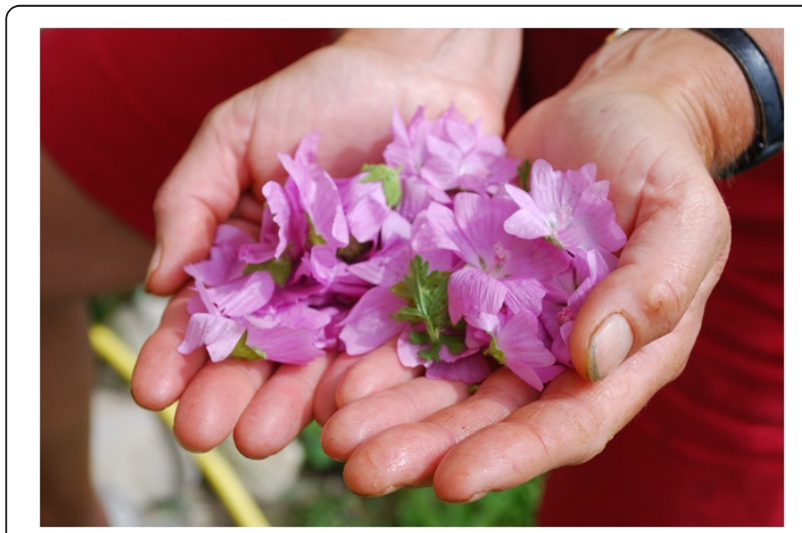

Figure 8 Malva sylvestris for herbal tea (Photo: Susanne Grasser). cultural heritage. The request is that the Biosphere Reserve's management keeps supporting initiatives like this to reinforce people's awareness of the value of their own local knowledge, their experience and practice and the appreciation of their own habits as an expression of their regional identity.

\section{Competing interests \\ The authors declare that they have no competing interests.}

\section{Authors' contributions}

SG designed the methods approach, carried out field work, composed the literature review and drafted the manuscript. CS conducted quantitative data analysis and supplemented the draft. CRV assisted greatly in all stages of this study. All authors read and approved the final manuscript.

\section{Acknowledgements}

The authors would first like to thank the people of Grosses Walsertal who shared their knowledge with them. They would especially like to thank the people who run the Bergtee association, Elisabeth and Hanno Burtscher and Ilga Bickel, who opened up their homes and offered insight into their practice. They are also indebted to the Austrian Academy of Sciences which funds this project under the Man and Biosphere Programme (project title: "Monitoring of Biocultural Diversity in the Biosphere Reserve "Grosses Walsertal"Norarlberg, Austria - The use and management of biodiversity of crops, cultivars and wild gathered plant species").

Received: 21 December 2011 Accepted: 25 July 2012

Published: 13 August 2012

\section{References}

1. Cunningham AB: Applied ethnobotany. People, wild plant use and conservation. 1 publth edition. London u.a: Earthscan; 2001.

2. Plenderleith K: Traditional Agricultural and Soil Management. In: UNEP: Cultural and Spiritual Values of Biodiversity. London: Intermediate Technology Publications; 1999:285-323.

3. Ertuğ F: An ethnobotanical study in Central Anatolia (Turkey). Econ Bot 2000, 54:155-182.

4. Forbes MHC: Gathering in the Argolid: a Subsistence Subsystem in a Greek Agricultural Community. In Regional Variation in Modern Greece and Cyprus: Toward a Perspective on the Ethnography of Greece. Annals of the New York Academy of Sciences, Volume 268. Edited by Dimen M, Friedl E; 1976:251-264.

5. Pieroni A: Gathered wild food plants in the upper valley of the Serchio river (Garfagnana), central Italy. Econ Bot 1999, 53:327-341.

6. Nebel S, Pieroni A, Heinrich M: Ta chòrta: Wild edible greens used in the Graecanic area in Calabria, Southern Italy. Appetite 2006, 47:333-342

7. Bonet MÀ, Vallès J: Use of non-crop food vascular plants in Montseny biosphere reserve (Catalonia, Iberian Peninsula). Int J Food Sci Nutr 2002, 53:225-248

8. Pardo de Santayana M, Tardío J, Blanco E, Carvalho AM, Lastra JJ, San Miguel E, Morales R: Traditional knowledge of wild edible plants used in the northwest of the Iberian Peninsula (Spain and Portugal): A comparative study. J Ethnobiol Ethnomed 2007, 3:27.

9. Menendez-Baceta G, Aceituno-Mata L, TardÃo J, Reyes-GarcÃa V, Pardo-de-Santayana M: Wild edible plants traditionally gathered in Gorbeialdea (Biscay, Basque Country). Genet Resour Crop Evol 2011, 1-19. In press.

10. Della A, Paraskeva-Hadjichambi D, Hadjichambis AC: An ethnobotanical survey of wild edible plants of Paphos and Larnaca countryside of Cyprus. J Ethnobiol Ethnomed 2006, 2:34.

11. Jarić S, Popović Z, Mačukanović-Jocić M, Djurdjević L, Mijatović M, Karadžić B, Mitrović M, Pavlović P: An ethnobotanical study on the usage of wild medicinal herbs from Kopaonik Mountain (Central Serbia). J Ethnopharmacol 2007, 111:160-175.

12. Menković N, Savikin K, Tasić S, Zdunić G, Stešević D, Milosavljević S, Vincek D: Ethnobotanical study on traditional uses of wild medicinal plants in Prokletije Mountains (Montenegro). J Ethnopharmacol 2011, 133:97-107. 
13. Mustafa B, Hajdari A, Pajazita Q, Syla B, Quave CL, Pieroni A: An ethnobotanical survey of the Gollak region, Kosovo. Genet Resour Crop Evol 2011, 1-16. In press.

14. Sarić-Kundalić B, Dobeš C, Klatte-Asselmeyer V, Saukel J: Ethnobotanical survey of traditionally used plants in human therapy of east, north and north-east Bosnia and Herzegovina. J Ethnopharmacol 2011, 133:1051-1076.

15. Sarić-Kundalić B, Dobeš C, Klatte-Asselmeyer V, Saukel J: Ethnobotanical study on medicinal use of wild and cultivated plants in middle, south and west Bosnia and Herzegovina. J Ethnopharmacol 2010, 131:33-55.

16. Pieroni A, Gray C: Herbal and food folk medicines of the Russlanddeutschen living in Künzelsau/Taläcker, South-Western Germany. Phytother Res 2008, 22:889-901.

17. Christanell A, Vogl-Lukasser B, Vogl CR, Gütler M: The Cultural Significance of Wild-gathered Plant Species in Kartitsch (Eastern Tyrol, Austria) and the Influence of Socioeconomic Changes on Local Gathering Practices. In Ethnobotany in the New Europe: people, health and wild plant resources. 1 publth edition. Edited by Pardo De Santayana M, Pieroni A, Puri R. New York, NY u.a: Berghahn Books; 2010:51-75.

18. Schunko C, Vogl CR: Organic farmers use of wild food plants and fungi in a hilly area in Styria (Austria). J Ethnobiol Ethnomed 2010, 6:17.

19. Brüschweiler S: Plantes et Savoirs des Alpes: L'exemple du val d'Anniviers. SA: Editions Monographic; 1999.

20. Luczaj L, Szymański WM: Wild vascular plants gathered for consumption in the Polish countryside: A review. J Ethnobiol Ethnomed 2007, 3:17.

21. Pieroni A, Giusti ME, Münz H, Lenzarini C, Turković G, Turković A: Ethnobotanical knowledge of the Istro-Romanians of Žejane in Croatia. Fitoterapia 2003, 74:710-719.

22. Tardío J, Pascual H, Morales R: Wild food plants traditionally used in the province of Madrid, Central Spain. Econ Bot 2005, 59:122-136.

23. Pardo de Santayana M: Ethnobotany in the new Europe. People, health and wild plant resources. 1 publth edition. New York, NY u.a: Berghahn Books; 2010.

24. Pieroni A: Medicinal plants and food medicines in the folk traditions of the upper Lucca Province, Italy. J Ethnopharmacol 2000, 70:235-273.

25. Signorini MA, Piredda M, Bruschi P: Plants and traditional knowledge: An ethnobotanical investigation on Monte Ortobene (Nuoro, Sardinia). J Ethnobiol Ethnomed 2009, 5:6.

26. González-Tejero MR, Casares-Porcel M, Sánchez-Rojas CP, Ramiro-Gutiérrez JM, Molero-Mesa J, Pieroni A, Giusti ME, Censorii E, de Pasquale C, Della A, et al: Medicinal plants in the Mediterranean area: Synthesis of the results of the project Rubia. J Ethnopharmacol 2008, 116:341-357.

27. Ghirardini MP, Carli M, del Vecchio N, Rovati A, Cova O, Valigi F, Agnetti G, Macconi M, Adamo D, Traina M, et al: The importance of a taste. A comparative study on wild food plant consumption in twenty-one local communities in Italy. J Ethnobiol Ethnomed 2007, 3:22.

28. Machatschek M: Nahrhafte Landschaft. Mädesüß, Austernpilz, Bärlauch, Gundelrebe, Meisterwurz, Schneerose, Walnuss, Zirbe und andere wiederentdeckte Nutz- und Heilpflanzen. Weimar: Böhlau Verlag Wien Köln; 2004.

29. Machatschek M: Nahrhafte Landschaft. Ampfer, Kümmel, Wildspargel, Rapunzelgemüse, Speiselaub und andere wiederentdeckte Nutz- und Heilpflanzen. 3, unveränd. Auflth edition. Weimar: Böhlau Verlag Wien Köln; 2007.

30. Schertler R: Vorarlberger Kräuterwelten. Ein botanischer Streifzug durchs Ländle. Innsbruck, Austria: Loewenzahn Verlag; 2005.

31. Helm EM: Feld-, Wald- und Wiesenkochbuch: Sammeln. Zubereiten und Einkochen von Wildgemüsen und Wildfüchten München: Heyne; 1982.

32. Pieroni A: Local plant resources in the ethnobotany of Theth, a village in the Northern Albanian Alps. Genet Resour Crop Evol 2008, 55:1197-1214.

33. Pardo de Santayana M, Pieroni A, Puri RK (Eds): Ethnobotany in the new Europe. People, health and wild plant resources, Volume 1. publ. New York, NY u.a: Berghahn Books; 2010.

34. Bonet MA, Parada M, Selga A, Vallès J: Studies on pharmaceutical ethnobotany in the regions of L'Alt Emporda and Les Guilleries (Catalonia, Iberian Peninsula). J Ethnopharmacol 1999, 68:145-168.

35. Raja D, Blanché C, Xirau JV: Contribution to the knowledge of the pharmaceutical ethnobotany of La Segarra region (Catalonia, Iberian Peninsula). J Ethnopharmacol 1997, 57:149-160.

36. Maffi L, Woodley E: Biocultural diversity conservation. A global sourcebook. 1 publth edition. London u.a: Earthscan; 2010.
37. Heinrich M, Leonti M, Nebel S, Peschel W: "Local food - Nutraceuticals": An example of a multidisciplinary research project on local knowledge. J Physiol Pharmacol 2005, 56:5-22.

38. Nebel $\mathrm{S}$, Heinrich M: Ta chòrta: A comparative ethnobotanical-linguistic study of wild food plants in a graecanic area in Calabria, Southern Italy. Econ Bot 2009, 63:78-92.

39. Heinrich M: Local mediterranean food plants and nutraceuticals. Basel u.a.: Karger; 2006.

40. UNESCO: The Sevilla Strategy for biosphere reserves, Series. Nature and Resources 1995, 31(2):2-17. UNESCO (ed.).

41. Leitbild BSR GWT: Leitbild Biosphärenpark Großes Walsertal. Überarbeitete Kurzfassung (von 1999). EMAS - Umwelt- und Nachhaltigkeitspolitik. Thüringerberg, Vorarlberg: Biosphärenparkbüro der Regionalplanungsgemeinschaft Großes Walsertal; 2004:18.

42. Bridgewater PB: Biosphere reserves: Special places for people and nature. Environ Sci Policy 2002, 5:9-12.

43. Biosphärenpark Großes Walsertal. 2011 http://www.grosseswalsertal.at/.

44. Reutz-Hornsteiner B: Case Study of the Biosphere Reserve Grosses Walsertal. In EUROMAB Austria 2005: Meeting of the EuroMAB Biosphere Reserve Coordinators and Managers. Edited by Österreichische UNESCO Kommission. 2006:58-59.

45. Werner R: Niederschlag und Gewitter. Verdunstung, Luftdruck, Wind: Schnee und Gletscher; 2001

46. Werner R: Klima von Vorarlberg Band I. Bodentemperatur, Wassertemperatur, Luftfeuchte, Bewölkung, Nebel: Lufttemperatur; 2001.

47. Burger-Scheidlin $\mathrm{H}$ : Wetter- und Klimakonzepte von Landwirten. Wahrnehmung und Wissen zwischen Praxis und Theorie; eine sozialanthropologisch - ethnoklimatologische Studie im Großen Walsertal, Österreich. Dissertation. Vienna: University of Vienna; 2007.

48. Dobler E: Vom Ackerbau im Großen Walsertal. In: Walserheimat in Vorarlberg, Volume 15; 1974:188-199.

49. Bodlak S: Alti Pflenk vo da Krüter. Heimat 1923, 178-182.

50. Künzle J: Chrut und Uchrut. Verbesserte Auflage 525.000-535.000. Feldkirch: Verlag für Deutschland und Österreich: F. Unterberger; 1930.

51. Bertel F, Burtscher E, Feßler E, Türtscher M: We' ma-n an Wehtig hed saäd ma sött ma. 2. Auflage edn. Eigenverlag: Fontallea; 1996.

52. Eco_Monte: Bericht zur Ist-Analyse. Energieinstitut Vorarlberg: Konstanz und Bregenz: Ökologieinstitut; 2002:78.

53. GWT Bischof M: Dossier on Grosses Walsertal Biosphere Park - winning region in the EDEN AWARDS 2009. Thüringerberg, Austria: Grosses Walsertal Tourism Association; 2009.

54. Moser R: Periodoc review. UNESCO Biosphere Reserve Grosses Walsertal. Thüringerberg, Austria: Biosphere Reserve Großes Walsertal; 2010.

55. Weller SC, Romney AK: Systematic data collection. Newbury Park, Calif. u.a: Sage Publ; 1988

56. Bernard HR: Research methods in anthropology. Qualitative and quantitative methods. 3rd edition. Walnut Creek, Calif. u.a: AltaMira Press; 2002.

57. International Society of Ethnobiology Code of Ethics (with 2008 additions): http://ethnobiology.net/code-of-ethics/ http://ethnobiology.net/code-ofethics/.

58. Lauber K, Wagner G: Flora Helvetica. 4. Aufl. 4. Aufl. edn. Haupt Verlag: Bern, Stuttgart, Wien; 2007.

59. Microsoft: Microsoft Access 2007. Redmont: Microsoft corporation; 2007.

60. Microsoft: Microsoft Excel 2007. Redmont: Microsoft corporation; 2007.

61. Borgatti SP, Everett MG, Freeman LC: UCINET for Windows: Software for Social Network Analysis. MAAnalytic Technologies: Harvard; 2002.

62. Express Scribe Transcription Software. Retrieved 09th September 2011. http://www.nch.com.au/scribe/index.html. http://www.nch.com.au/scribe/ index.html.

63. Teutsch JN (Ed): Vorarlberger Schreib-Kalender (Bregenzer Kalender) für das gemeine Jahr nach der gnadenreichen Geburt unseres Heilandes Jesu Christi. Bregenz, Austria: Verlag J.N. Teutsch; 2009.

64. Stepp JR, Moerman DE: The importance of weeds in ethnopharmacology. J Ethnopharmacol 2001, 75:19-23.

65. Pieroni A, Giusti ME: Alpine ethnobotany in Italy: Traditional knowledge of gastronomic and medicinal plants among the Occitans of the upper Varaita valley, Piedmont. J Ethnobiol Ethnomed 2009, 5:32.

66. Ploetz K, Orr B: Wild herb use in Bulgaria. Econ Bot 2004, 58:231-241.

67. Pardo de Santayana M, Blanco E, Morales R: Plants known as té in Spain: An ethno-pharmaco-botanical review. J Ethnopharmacol 2005, 98:1-19. 
68. Agelet A, Vallès J: Studies on pharmaceutical ethnobotany in the region of Pallars (Pyrenees, Catalonia, Iberian Peninsula). Part III. Medicinal uses of non-vascular plants. J Ethnopharmacol 2003, 84:229-234.

69. Rigat $\mathrm{M}$, Bonet MA, Garcia S, Garnatje T, Vallès J: Studies on pharmaceutical ethnobotany in the high river Ter valley (Pyrenees, Catalonia, Iberian Peninsula). J Ethnopharmacol 2007, 113:267-277.

70. Viegi L, Pieroni A, Guarrera PM, Vangelisti R: A review of plants used in folk veterinary medicine in Italy as basis for a databank. J Ethnopharmacol 2003, 89:221-244

71. Agelet $A$, Vallès J: Studies on pharmaceutical ethnobotany in the region of Pallars (Pyrenees, Catalonia, Iberian Peninsula). Part I. General results and new or very rare medicinal plants. J Ethnopharmacol 2001, 77:57-70.

72. Ali-Shtayeh MS, Jamous RM, Al-Shafie JH, Elgharabah WA, Kherfan FA, Qarariah KH, Khdair IS, Soos IM, Musleh AA, Isa BA, et al: Traditional knowledge of wild edible plants used in Palestine (Northern West Bank): A comparative study. J Ethnobiol Ethnomed 2008, 4:13.

73. Etkin NL: Eating on the wild side: The pharmacologic, ecologic and social implications of using noncultigens. Tucson, Ariz: Univ. of Arizona Pr; 1994.

74. Guarrera PM, Lucia LM: Ethnobotanical remarks on central and southern Italy. J Ethnobiol Ethnomed 2007, 3:23.

75. Pardo de Santayana M, Tardío J, Morales R: The gathering and consumption of wild edible plants in the Campoo (Cantabria, Spain). Int J Food Sci Nutr 2005, 56:529-542.

76. Reyes-García V, Vila S, Aceituno-Mata L, Calvet-Mir L, Garnatje T, Jesch A Lastra JJ, Parada M, Rigat M, Vallès J, Pardo-de-Santayana M: Gendered Homegardens: A Study in Three Mountain Areas of the Iberian Peninsula. Econ Bot 2010, 64:235-247.

doi:10.1186/1746-4269-8-31

Cite this article as: Grasser et al.: Gathering "tea" - from necessity to connectedness with nature. Local knowledge about wild plant gathering in the Biosphere Reserve Grosses Walsertal (Austria). Journal of Ethnobiology and Ethnomedicine 2012 8:31.

\section{Submit your next manuscript to BioMed Central and take full advantage of:}

- Convenient online submission

- Thorough peer review

- No space constraints or color figure charges

- Immediate publication on acceptance

- Inclusion in PubMed, CAS, Scopus and Google Scholar

- Research which is freely available for redistribution 